\title{
Membrane lipids: where they are and how they behave
}

\section{Gerrit van Meer*, Dennis R. Voelker ${ }^{\ddagger}$ and Gerald W. Feigenson $\S$}

Abstract | Throughout the biological world, a 30 A hydrophobic film typically delimits the environments that serve as the margin between life and death for individual cells.

Biochemical and biophysical findings have provided a detailed model of the composition and structure of membranes, which includes levels of dynamic organization both across the lipid bilayer (lipid asymmetry) and in the lateral dimension (lipid domains) of membranes. How do cells apply anabolic and catabolic enzymes, translocases and transporters, plus the intrinsic physical phase behaviour of lipids and their interactions with membrane proteins, to create the unique compositions and multiple functionalities of their individual membranes?

\section{Triacylglycerol}

A family of storage lipids

consisting of glycerol esterified

to three fatty acids, forming the

hydrophobic core of lipid

droplets and blood

lipoproteins together with

steryl esters.

Steryl ester

A family of storage lipids

consisting of sterol esterified to one fatty acid, forming the

hydrophobic core of lipid droplets and blood

lipoproteins together with triacylglycerol molecules.

\section{Amphipathic}

The characteristic of being polar on one side of the molecule and apolar on the opposite side.

* Bijvoet Center and Institute of Biomembranes, Utrecht University, 3584 CH Utrecht, The Netherlands.

${ }^{\ddagger}$ Department of Medicine, National Jewish Medical Research Center, Denver, Colorado 80206, USA.

§Department of Molecular Biology and Genetics, Cornell University, Ithaca, New York 14853, USA.

Correspondence to G.v.M. e-mail:g.vanmeer@uu.nI doi: $10.1038 / \mathrm{nrm} 2330$
From the ongoing cataloguing of lipid structures (lipidomics), it is clear that eukaryotic cells invest substantial resources in generating thousands of different lipids ${ }^{1}$. Why do cells use $\sim 5 \%$ of their genes to synthesize all of these lipids? The fundamental biological maxim that 'structure subserves function' implies that there must be evolutionary advantages that are dependent on a complex lipid repertoire. Although we now understand the specific functions of numerous lipids, the full definition of the utility of the eukaryotic lipid repertoire remains elusive.

Lipids fulfil three general functions. First, because of their relatively reduced state, lipids are used for energy storage, principally as triacylglycerol and steryl esters, in lipid droplets. These function primarily as anhydrous reservoirs for the efficient storage of caloric reserves and as caches of fatty acid and sterol components that are needed for membrane biogenesis. Second, the matrix of cellular membranes is formed by polar lipids, which consist of a hydrophobic and a hydrophilic portion. The propensity of the hydrophobic moieties to self-associate (entropically driven by water), and the tendency of the hydrophilic moieties to interact with aqueous environments and with each other, is the physical basis of the spontaneous formation of membranes. This fundamental principle of amphipathic lipids is a chemical property that enabled the first cells to segregate their internal constituents from the external environment. This same principle is recapitulated within the cell to produce discrete organelles. This compartmentalization enables segregation of specific chemical reactions for the purposes of increased biochemical efficiency and restricted dissemination of reaction products. In addition to the barrier function, lipids provide membranes with the potential for budding, tubulation, fission and fusion, characteristics that are essential for cell division, biological reproduction and intracellular membrane trafficking. Lipids also allow particular proteins in membranes to aggregate, and others to disperse. Finally, lipids can act as first and second messengers in signal transduction and molecular recognition processes. The degradation of amphipathic lipids allows for bipartite signalling phenomena, which can be transmitted within a membrane by hydrophobic portions of the molecule and also propagated through the cytosol by soluble (polar) portions of the molecule. In addition, some lipids function to define membrane domains, which recruit proteins from the cytosol that subsequently organize secondary signalling or effector complexes.

Progress in biophysics, chemistry and genetics has attracted renewed attention to the biological roles of the great variety of membrane lipids. One property of lipids that has fascinated scientists is their phase behaviour. In cells, lipids can adopt various fluid and solid phases, which are characterized by a different spatial arrangement and motional freedom of each lipid with respect to its neighbours. Biophysical approaches have defined the principles of coexistence of two fluid phases (with different physical characteristics within a single membrane plane) that are delimited by a phase boundary, and the consequences on membrane organization ${ }^{2,3}$. Advances in mass spectrometry have catalysed precipitous developments in holistic, high-sensitivity (and high-throughput) lipid analysis, welcoming lipids to the realm of genomics and proteomics. The identification of new disease-related genes has revealed the involvement of lipid-related proteins, such as enzymes (lysosomal hydrolases), transporters (ATPbinding cassette $(\mathrm{ABC})$ transporters) or lipid binding 

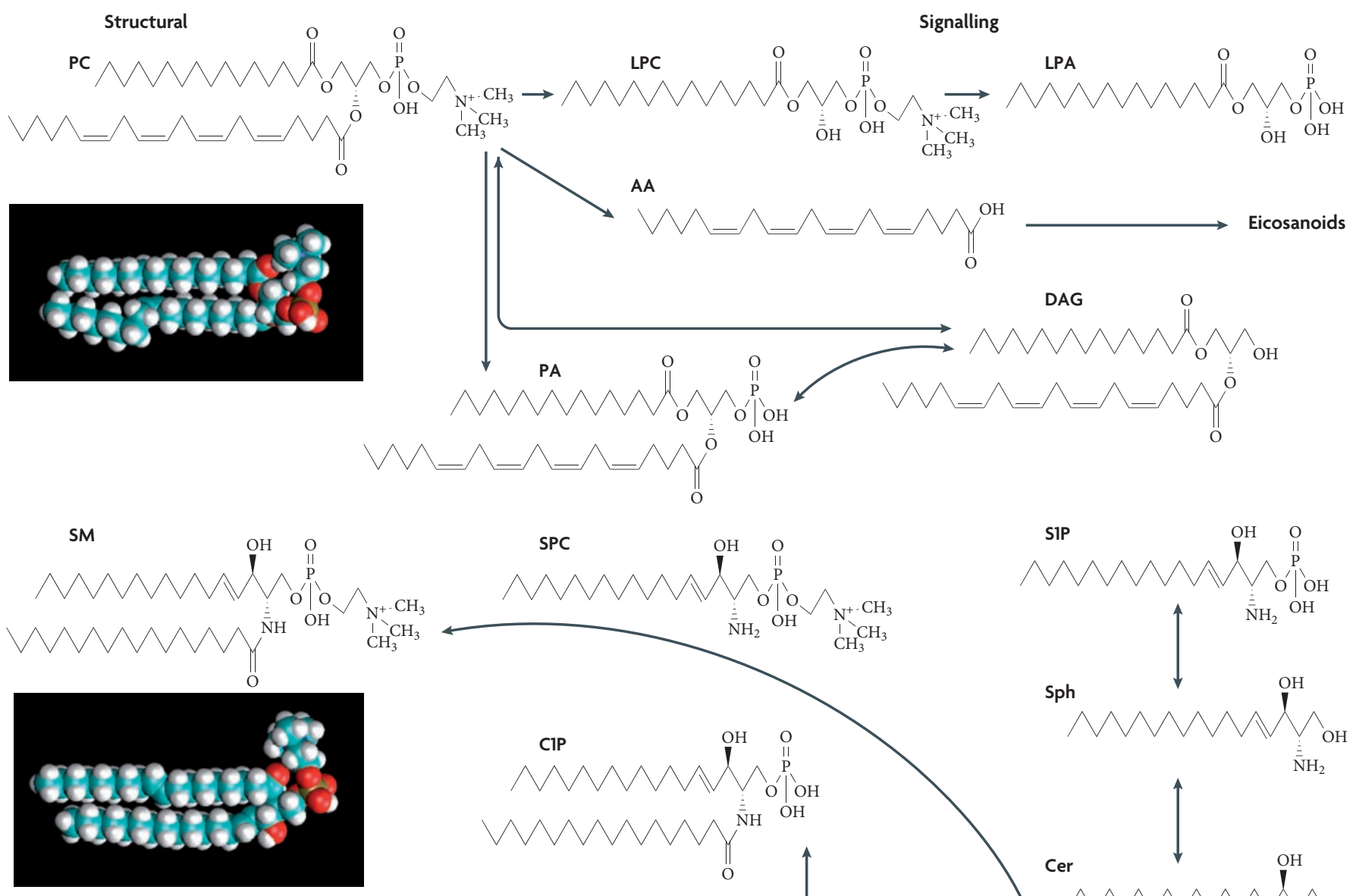

SPC
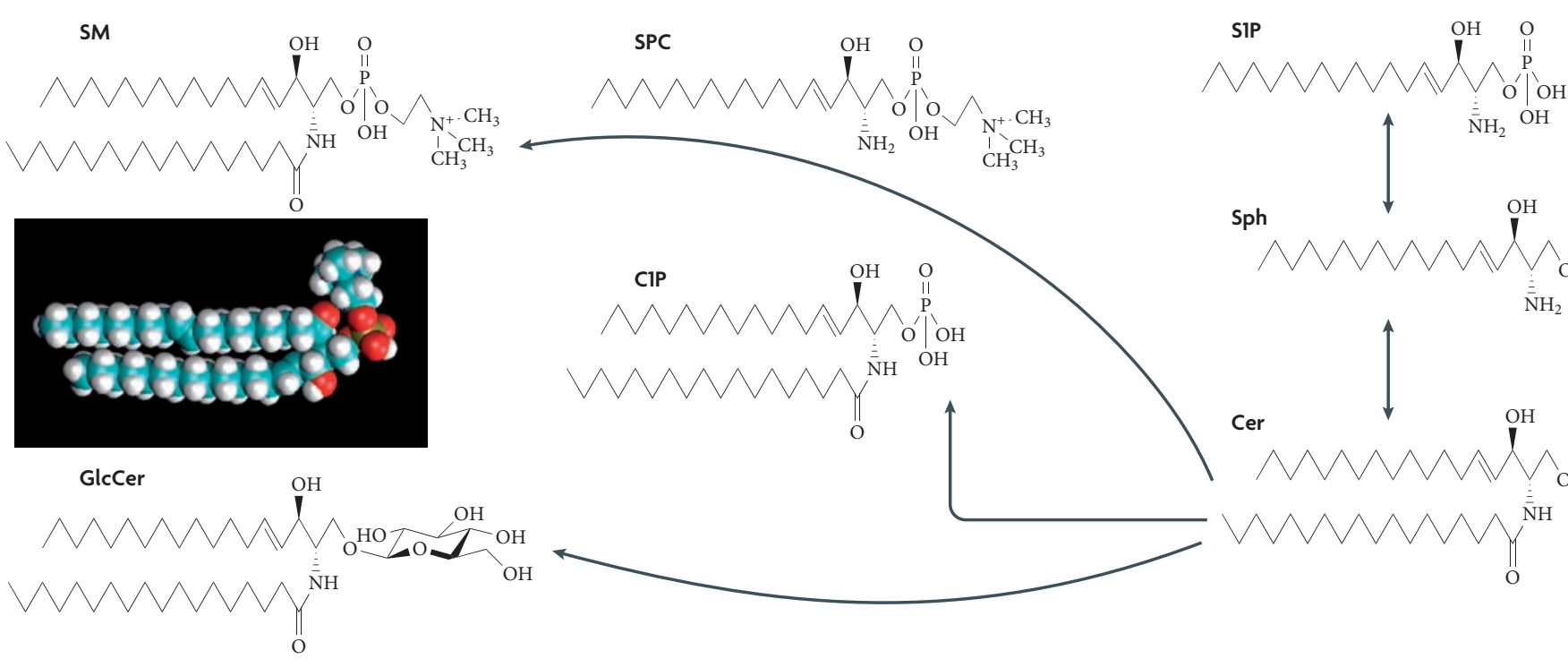

Sph
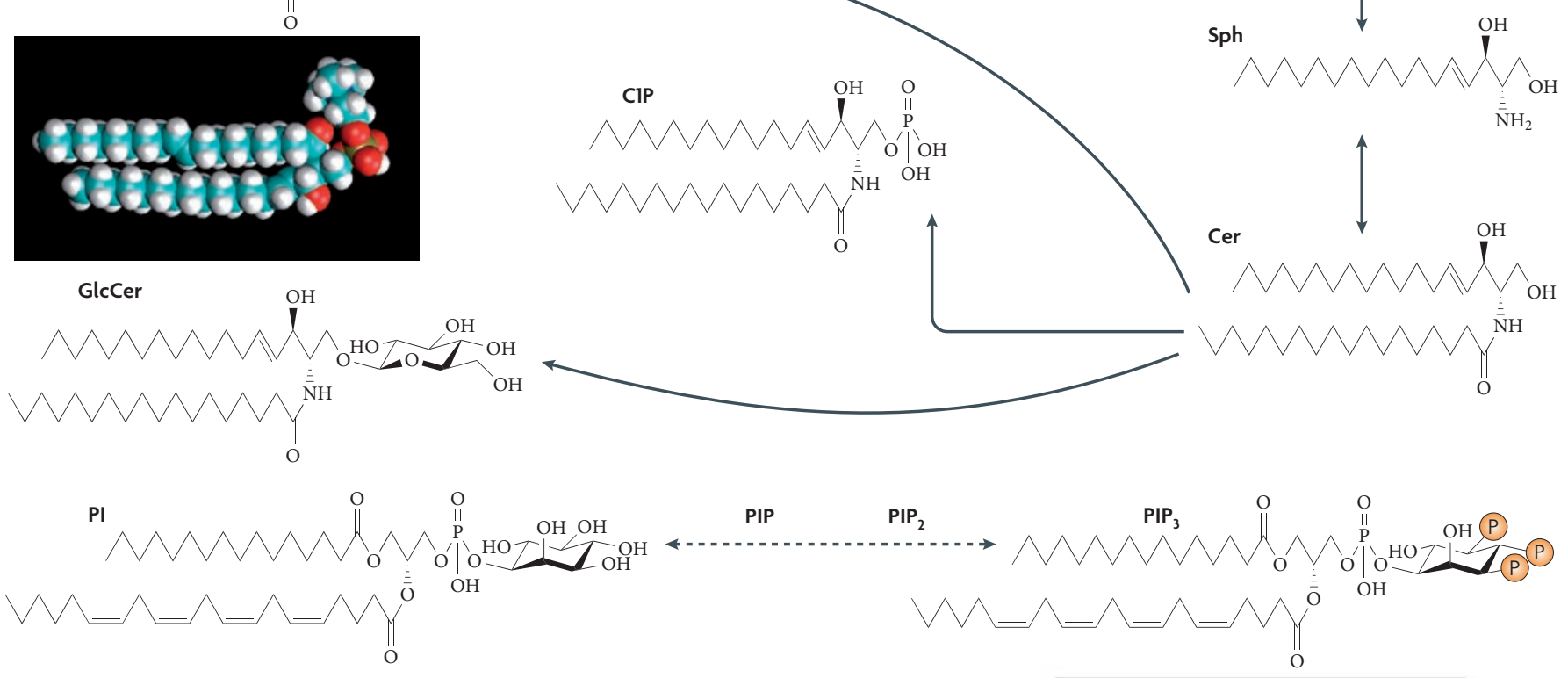

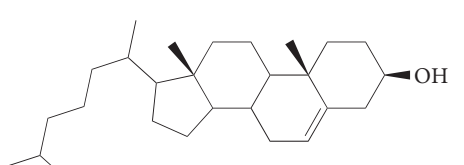

Cholesterol

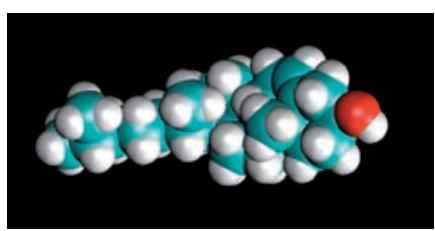

Figure 1 | Membrane lipids and lipid second messengers. The main eukaryotic membrane lipids are the glycerophospholipids such as phosphatidylcholine (PtdCho; PC). Their diacylglycerol (DAG) backbone carries a phosphate (phosphatidic acid; PA) esterified to either a choline (forming PtdCho), ethanolamine (forming phosphatidylethanolamine (PtdEtn); not shown), serine (forming phosphatidylserine (PtdSer); not shown), or inositol (forming phosphatidylinositol (Ptdlns); PI). The prototypical phospholipid, dipalmitoyl-PtdCho, exhibits nearly cylindrical molecular geometry with a cross-sectional surface area of $64 \AA^{2}$ and a head-to-tail length of $19 \AA$ (REF. 122). The phosphosphingolipid sphingomyelin (SM) and the glycosphingolipid glucosylceramide (GlcCer) have a ceramide (Cer) backbone, consisting of a sphingoid base (such as sphingosine; Sph), which

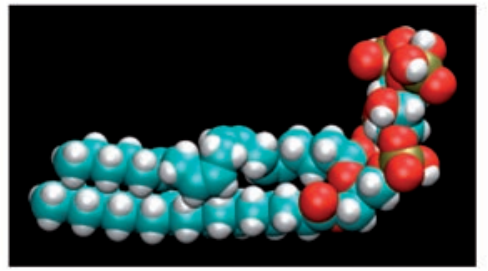

is amide-linked to a fatty acid. Yeast sphingolipids carry a C26 fatty acid and have phosphoinositol-X substituents that contain additional mannoses and phosphates. Breakdown products of membrane lipids serve as lipid second messengers. The glycerolipid-derived signalling molecules include lysoPtdCho (LPC), lysoPA (LPA), PA and DAG. The sphingolipid-derived signalling molecules include sphingosylphosphorylcholine (SPC), Sph, sphingosine-1-phosphate (S1P), Cer-1-phosphate (C1P) and Cer. Arachidonic acid (AA) yields the signalling eicosanoids and endocannabinoids (not shown). The various phosphorylated Ptdlns molecules (PIPs; also known as the phosphoinositides) mark cellular membranes and recruit cytosolic proteins. They are interconverted by the actions of kinases and phosphatases. Fig. modified with permission from REF. 5 @ (2005) Macmillan Publishers Ltd. 
proteins (START domain proteins). It is now essential to define the molecular function of the corresponding gene products to improve diagnostics and develop cures for the respective diseases. Pathological processes such as viral infection have been unexpectedly connected to specific membrane lipids and their biophysical properties ${ }^{4}$, emphasizing the need for an integrated study of cellular lipids using lipidomic methods 5 .

Here, we introduce the main lipids of biomembranes, their topology and the spatial organization of their metabolism. We emphasize how metabolism and selective transport maintain the differences in lipid composition between organelles, and highlight how the biophysical properties of the different lipids and their phase behaviour contribute to membrane function. We discuss mammalian cells and yeast to highlight similarities, and demonstrate how cells use different chemical structures with similar physical properties for common purposes.

\section{The repertoire of membrane lipids}

The major structural lipids in eukaryotic membranes are the glycerophospholipids: phosphatidylcholine (PtdCho), phosphatidylethanolamine (PtdEtn), phosphatidylserine (PtdSer), phosphatidylinositol (PtdIns) and phosphatidic acid (PA). Their hydrophobic portion is a diacylglycerol (DAG), which contains saturated or cis-unsaturated fatty acyl chains of varying lengths (FIG. 1). PtdCho accounts for $>50 \%$ of the phospholipids in most eukaryotic membranes. It self-organizes spontaneously as a planar bilayer in which each PtdCho has a nearly cylindrical molecular geometry, with the lipidic tails facing each other and the polar headgroups interfacing with the aqueous phase. Most PtdCho molecules have one cis-unsaturated fatty acyl chain, which renders them fluid at room temperature. PtdEtn assumes a conical molecular geometry because of the relatively small size of its polar headgroup. The inclusion of PtdEtn in PtdCho bilayers imposes a curvature stress onto the membrane, which is used for budding, fission and fusion ${ }^{6}$. Non-bilayer lipids like PtdEtn and cardiolipin (CL) may also be used to accommodate membrane proteins and modulate their activities ${ }^{6,7}$. An additional factor that contributes to curvature stress in biomembranes is the asymmetric distribution of various lipids between the two bilayer leaflets.

The sphingolipids constitute another class of struc-

Lysosomal hydrolase An enzyme that hydrolyses biomolecules in lysosomes.

Dolichol

A family of long-chain, unsaturated and methylated hydrocarbons that consist of varying numbers of isoprene units that terminate in an $\alpha$-saturated isoprenoid group and contain an alcohol functional group.

Dolicholphosphate and dolicholpyrophosphate anchor

sugar molecules to the ER membrane for transfer to proteins in the ER lumen. tural lipids. Their hydrophobic backbone is ceramide (Cer). The major sphingolipids in mammalian cells are sphingomyelin (SM) and the glycosphingolipids (GSLs), which contain mono-, di- or oligosaccharides based on glucosylceramide (GlcCer) and sometimes galactosylceramide $(\mathrm{GalCer})^{8}$. Gangliosides are GSLs with terminal sialic acids. Sphingolipids have saturated (or trans-unsaturated) tails so they are able to form taller, narrower cylinders than PtdCho lipids of the same chain length and they pack more tightly, adopting the solid 'gel' or $\mathrm{s}_{\mathrm{o}}$ phase; they are fluidized by sterols (BOX 1). Sterols are the major non-polar lipids of cell membranes: cholesterol predominates in mammals whereas ergosterol predominates in yeast. According to the umbrella model ${ }^{9,10}$, the preferential mixing of sterols with sphingolipids is caused by shielding of the non-polar sterol by the sphingolipid headgroup rather than being caused by preferential intermolecular interactions (see the review by Ikonen in this issue).

Signalling-induced hydrolysis of glycerolipids and sphingolipids produces parallel series of messenger lipids: lysoPtdCho (LPC), lysoPA (LPA), PA and DAG, versus sphingosylphosphorylcholine (SPC), sphingosine (Sph), sphingosine-1-phosphate (S1P), ceramide-1-phosphate (C1P) and Cer (FIG. 1). LPC, LPA, SPC, Sph and S1P carry only one aliphatic chain and readily leave membranes; they signal through related membrane receptors ${ }^{11}$. By contrast, PA, DAG, C1P and Cer remain in the membrane and can recruit cytosolic proteins ${ }^{12}$. Interestingly, when signalling lipids such as Cer are generated in large quantities ${ }^{13,14}$, they can also affect membrane physical properties such as lipid phase behaviour. Cer can displace cholesterol from lipid umbrellas ${ }^{15}$ and drive its esterification. Phosphorylated derivatives of PtdIns (FIG. 1) participate in signalling and recognition. These phosphoinositides are important in defining organelle identity (FIG. 2) and in recruiting both soluble and membrane proteins to specific membranes.

\section{Lipid metabolism and localization}

The variation in headgroups and aliphatic chains allows the existence of $>1,000$ different lipid species in any eukaryotic cell ${ }^{1}$. Interestingly, the phospholipids and sterols are not distributed homogeneously throughout the main organelles of mammals and yeast (FIG. 2). Within eukaryotic cells, the synthesis of structural lipids is geographically restricted. Local lipid metabolism is the first determinant of the unique compositions of organelles.

The ER: the main site of lipid synthesis. The main lipid biosynthetic organelle is the endoplasmic reticulum $(\mathrm{ER})^{16}$, which produces the bulk of the structural phospholipids and cholesterol (ergosterol in yeast) (FIG. 2), as well as significant levels of triacylglycerol and cholesteryl esters that have non-structural roles. The ER also produces Cer, which is the precursor for complex sphingolipids, and GalCer is produced in the ER of myelinating and epithelial cells, where it stabilizes myelin and apical membranes ${ }^{17}$. The mitochondria-associated membranes are a subfraction of the ER that cofractionates with mitochondria, to which the membranes are attached through contact sites. They are particularly enriched in specific lipid biosynthetic enzymes in both mammals and yeast ${ }^{18,19}$, suggesting that lipid synthesis may be subcompartmentalized in the ER.

Although the ER is the main site of cholesterol synthesis, this lipid is rapidly transported to other organelles. Indeed, the ER - which is situated at the beginning of the secretory pathway - displays only low concentrations of sterols and complex sphingolipids. The resulting loose packing of ER membrane lipids is consistent with the function of the organelle in the insertion and transport of newly synthesized lipids and proteins. The ER harbours minor lipids that function as both pathway intermediates and legitimate pathway endproducts (including DAG, cytidine diphosphoDAG, PA, lysophospholipids) and dolichol. 


\section{Box 1 | Lipid phases in membranes}

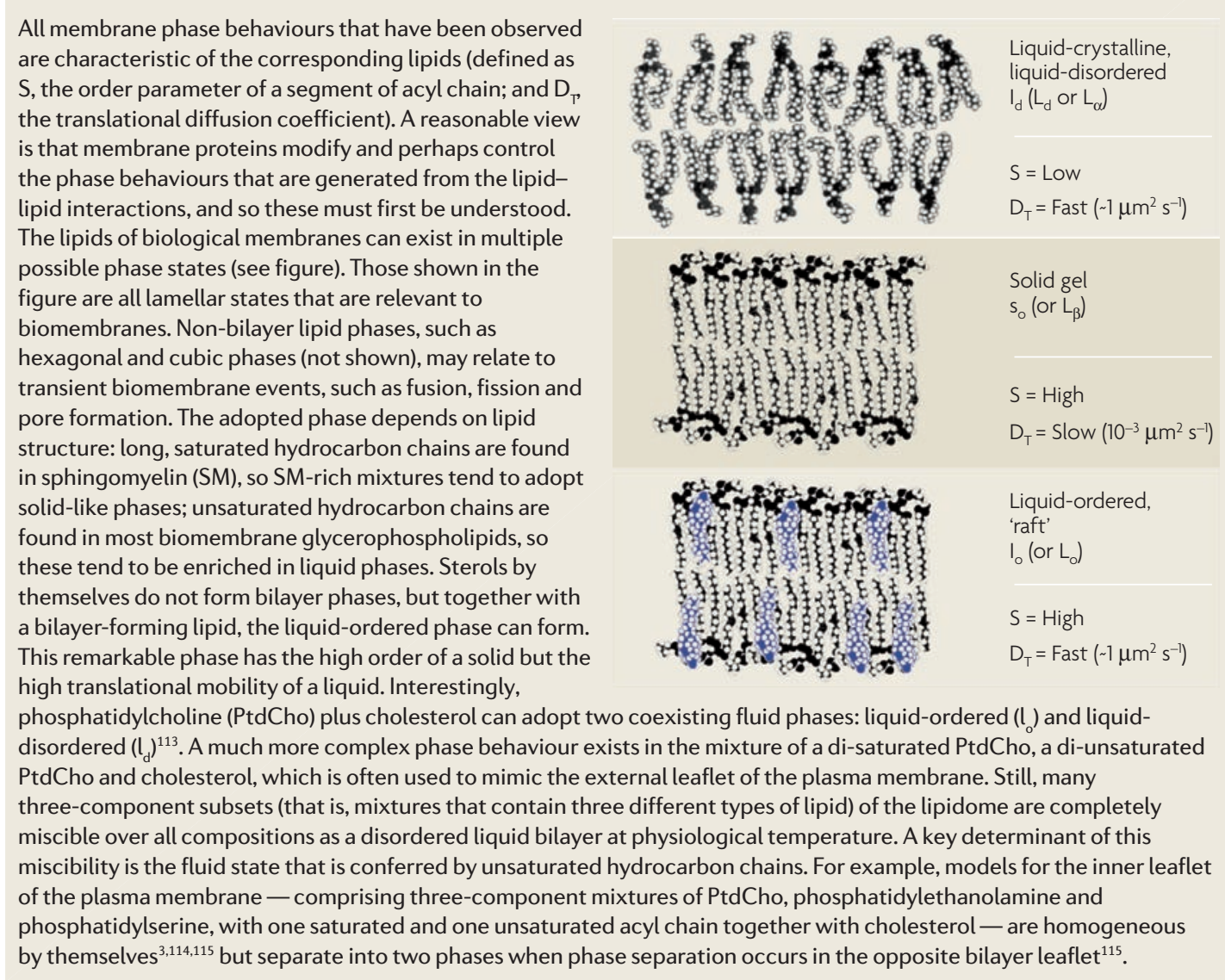

The Golgi apparatus: a lipid-based sorting station. Significant levels of lipid synthesis occur in the Golgi. The mammalian Golgi specializes in sphingolipid synthesis, and produces SM, GlcCer, lactosylceramide (LacCer) and higher-order GSLs ${ }^{20}$, all of which are primarily destined for export to the plasma membrane. The production of sphingolipids may have an important role in the sorting of membrane proteins and lipids between the ER, the plasma membrane and endosomes or the vacuole through lipid rafts (see below). The terminal step of PtdCho synthesis can occur by enzymes in both the ER and the Golgi ${ }^{21}$. The Golgi-specific cholinephosphotransferase may have a role in regulating secretory processes by controlling the levels of DAG in the organelle. The yeast Golgi is involved in synthesis of sphingolipids of the ceramide phosphoinositol (CerPIns-X; where $\mathrm{X}$ is $-\mathrm{H}$, mannose or mannose phosphoinositol) series ${ }^{20}$. It also synthesizes PtdEtn via a PtdSer decarboxylase ${ }^{22}$.

Multivesicular body An endosome containing internal vesicles that originate from inward budding. This direction of budding is away from the cytosol (opposite to the regular budding of transport vesicles) and a different molecular machinery has been found to be responsible.
The plasma membrane: built for stability. Plasma membranes are enriched in sphingolipids and sterols (FIC. 2), which are packed at a higher density than glycerolipids and resist mechanical stress. Although the plasma membrane is not involved in autonomous synthesis of its structural lipids, numerous reactions for either synthesizing or degrading lipids that are involved in signalling cascades have been described for the organelle $e^{23}$. The turnover of SM at the plasma membrane is high enough for (re)synthesis of SM from Cer by the plasma membrane sphingomyelin synthase SMS2 to contribute significantly to the total cellular SM level ${ }^{24,25}$.

Lipids and the endocytic pathway. Early endosomes are similar to plasma membranes, but on maturation to late endosomes there is a decrease in sterols and PtdSer and a dramatic increase in bis(monoacylglycero)phosphate $(\mathrm{BMP})^{26}$. BMP functions in multivesicular body generation, fusion processes and sphingolipid hydrolysis ${ }^{27,28}$. A dedicated system of kinases and phosphatases produces and hydrolyses specific phosphoinositides ${ }^{23}$, including PtdIns $(4,5) \mathrm{P}_{2}$ on plasma membranes, PtdIns3P on early endosomes, PtdIns $(3,5) \mathrm{P}_{2}$ on late endosomes and PtdIns4P on the (trans)-Golgi network (FICS 1,2). These phosphoinositides identify endocytic membranes and allow them to recruit proteins from the cytosol that are involved in vesicle trafficking and other aspects of cellular homeostasis ${ }^{23}$. Lipid mediators of signalling and recognition processes are numerous and work through specific protein-lipid interactions ${ }^{23}$. The levels of these signalling molecules are extremely small compared with the levels of bulk membrane lipids. Site-specific generation and turnover and combinatorial recognition are central principles of lipids as conveyors of information (see the review by Lemmon in this issue). It is a major challenge in membrane biology to elucidate how these systems are topologically and temporally regulated. 


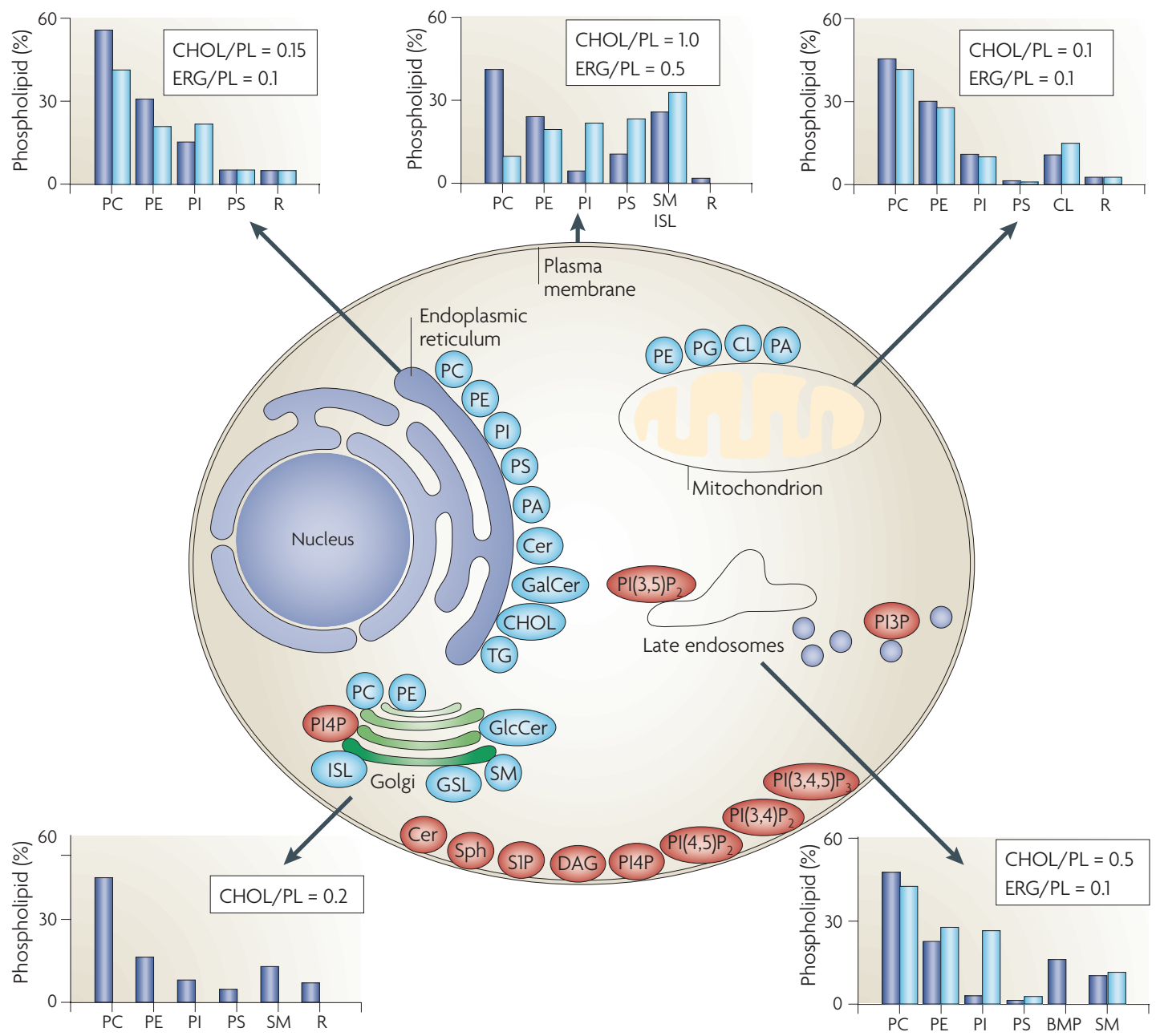

Figure 2 | Lipid synthesis and steady-state composition of cell membranes. The lipid composition of different membranes varies throughout the cell. The lipid compositional data (shown in graphs) are expressed as a percentage of the total phospholipid (PL) in mammals (blue) and yeast (light blue). As a measure of sterol content, the molar ratio of cholesterol (CHOL; in mammals) and ergosterol (ERG; in yeast) to phospholipid is also included. The figure shows the site of synthesis of the major phospholipids (blue) and lipids that are involved in signalling and organelle recognition pathways (red). It should be appreciated that the levels of signalling and recognition lipids are significantly below $1 \%$ of the total phospholipid, except for ceramide (Cer). The major glycerophospholipids assembled in the endoplasmic reticulum (ER) are phosphatidylcholine (PtdCho; PC), phosphatidylethanolamine (PtdEtn; PE), phosphatidylinositol (Ptdlns; PI), phosphatidylserine (PtdSer; PS) and phosphatidic acid (PA). In addition, the ER synthesizes Cer, galactosylceramide (GalCer), cholesterol and ergosterol. Both the ER and lipid droplets participate in steryl ester and triacylglycerol (TG) synthesis. The Golgi lumen is the site of synthesis of sphingomyelin (SM), complex glycosphingolipids (GSLs) and yeast inositol sphingolipid (ISL) synthesis. PtdCho is also synthesized in the Golgi, and may be coupled to protein secretion at the level of its diacylglycerol (DAG) precursor. Approximately $45 \%$ of the phospholipid in mitochondria (mostly PtdEtn, PA and cardiolipin $(\mathrm{CL})$ ) is autonomously synthesized by the organelle. BMP (bis(monoacylglycero)phosphate) is a major phospholipid in the inner membranes of late endosomes ${ }^{26}$ (not shown). PG, phosphatidylglycerol; PI $(3,5) \mathrm{P}_{2}$, phosphatidylinositol- $(3,5)$ bisphosphate; $\mathrm{PI}(4,5) \mathrm{P}_{2}$, phosphatidylinositol-(4,5)-bisphosphate; $\mathrm{PI}(3,4,5) \mathrm{P}_{3}$, phosphatidylinositol-(3,4,5)-trisphosphate; PI4P, phosphatidylinositol-4-phosphate; R, remaining lipids; S1P, sphingosine-1-phosphate; Sph, sphingosine.

Mitochondria have bacterial lipids. Significant levels of lipid synthesis occur in the mitochondria. Mitochondria synthesize $\mathrm{LPA}^{29}$, of which a substantial amount is used for triacylglycerol formation ${ }^{30}$. They also synthesize PA and phosphatidylglycerol (PtdGro), which is used for the synthesis of CL, a lipid that is unique to the mitochondria, as well as PtdEtn. Decarboxylation of PtdSer produces mitochondrial PtdEtn, which is exported to other organelles in both mammals and yeast ${ }^{31}$.
The presence of PtdGro and up to 25 mole percent of $\mathrm{CL}$ in the inner membrane $\mathrm{e}^{32}$, in addition to its high PtdEtn/PtdCho ratio, are reminiscent of the bacterial origin of this membrane and are probably required for oxidative phosphorylation. The sterol content of mitochondria is generally low, except for cells that are involved in steroid hormone synthesis, in which the mitochondria import and metabolize cholesterol in concert with the $\mathrm{ER}^{33}$. 


\section{Lipid dynamic transmembrane distribution}

The lipid compositions of individual membranes can be further stratified with respect to the orientation of the lipid to the cytosol. Whereas all lipids are symmetrically distributed between the two leaflets of the ER membrane bilayer, the Golgi, plasma and endosomal membranes display an asymmetric lipid distribution with SM and GSLs on the non-cytosolic (luminal side), with PtdSer and PtdEtn enriched in the cytosolic leaflet ${ }^{34,35}$. The asymmetric distribution of lipids has important functional consequences. For example, when exposed on the cell surface, PtdSer acts as a susceptibility signal for phagocytosis and as a propagation signal in blood coagulation. Furthermore, lipid translocation to the cytosolic leaflet causes a lipid quantity imbalance that can contribute to the membrane bending that is required for vesicle budding $^{36}$. Lipid asymmetry in membranes is a consequence of multiple factors, including the biophysical properties that dictate the ability of a lipid to cross the bilayer spontaneously, retentive mechanisms that trap lipids in one leaflet of the bilayer, and the presence of transporters that assist lipid translocation.

In model membranes of bilayer-forming lipids, the rate of spontaneous polar lipid movement between bilayer leaflets is slow (hours to days for PtdCho lipids ${ }^{37,38}$ ) and is governed by the size, charge and polarity of the headgroup. The half time $\left(t_{1 / 2}\right)$ for translocation is days for complex GSLs but seconds for $\mathrm{Cer}^{39}, \mathrm{DAG}^{40,41}$ and sterols ${ }^{42}$. The rapid transbilayer movement of Cer and DAG is unrelated to the concentration of these lipids in each leaflet of the bilayer because other membrane lipids and proteins can bias their affinity to a specific leaflet by trapping them within domains in that leaflet ${ }^{39}$. This may be the case for cholesterol, which is supposedly enriched in the noncytosolic leaflet of plasma membranes due to its high affinity for sphingolipids, although the actual data on the orientation of cholesterol are controversial ${ }^{34,35}$. Similarly, negatively charged lipids such as fatty acids, PtdGro and PA can rapidly flip when their charge is neutralized by low $\mathrm{pH}$, but they can effectively be accumulated on one side of the membrane by a $\mathrm{pH}$ gradient ${ }^{43}$.

Transporter-mediated mechanisms of translocation. Compelling evidence exists for intrinsic non-specific lipid transporters within membranes of high phospholipid synthetic capacity, such as the ER in mammals and the inner membrane in Gram-negative bacteria. These lipogenic membranes appear to have an intrinsic capacity for trans-

P-type ATPase

One of a family of membraneembedded transporters that share a phosphorylated intermediate as part of their reaction cycle. It includes the $\mathrm{Ca}^{2+}$ - and $\mathrm{Na}^{+} / \mathrm{K}^{+}$-ATPases and the $\mathrm{P} 4$ family of lipid flippases.

Scramblase

A mechanism (involving

incompletely characterized components) that allows

transmembrane movement of lipids and relaxation of lipid

asymmetry on cell stimulation. to a prenylpyrophosphate backbone) are apparently translocated from the cytosol across the ER and the bacterial inner membrane by dedicated transporters ${ }^{45,46}$.

For some lipids, an asymmetric distribution is accomplished by insertion of the lipid into only one membrane leaflet with continual retention of the lipid in this leaflet (such as for SM and GSLs within the Golgi). In other cases, such as for PtdSer and PtdEtn, the lipids are synthesized within one leaflet of the ER but freely equilibrate between leaflets. Subsequently, in other membranes (including the Golgi and plasma membranes), ATP-dependent aminophospholipid transporters selectively translocate these lipids from the luminal leaflet of the bilayer to the cytosolic leaflet $^{35,47}$ (FIG. 3). Several of these transporters belong to the P4 subfamily of P-type ATPases ${ }^{36}$. Mammalian cells readily transport PtdSer and PtdEtn that have been labelled with a fluorescent probe (NBD) from the outer to the inner leaflet of the plasma membrane bilayer ${ }^{35}$. Yeast transports NBD-PtdSer, NBD-PtdEtn and NBD-PtdCho, and genetic approaches have identified the gene products Dnf1 and Dnf2 as the responsible P4 ATPases ${ }^{48}$, which also require a second subunit, $\underline{\operatorname{Lem} 3}$, for activity. Recent studies now show that the same P4 ATPases transport lysoPtdEtn (LPE) and LPC as bona fide native phospholipid substrates across yeast plasma membranes ${ }^{49,50}$. The flux of LPE and LPC is extremely high and meets all of the requirements for synthesis of PtdEtn and PtdCho for cell growth. Therefore, one major uncertainty is the fidelity of fluorescent lipid analogues in comparison with the behaviour of native phospholipids (BOX 2). Additional yeast P4 ATPases, Dnf3 and Drs2, reside in the Golgi and contribute to phospholipid asymmetry at this location together with their second subunit Cdc50 (REF. 51). Some evidence suggests that Dnf3 and Drs2 cycle through the plasma membrane and partially suppress mutations that eliminate Dnf1 and Dnf2 ${ }^{52}$. Unexpectedly, deletion of these ATPases not only produced defects in NBD-lipid flipping but also inhibited vesicular traffic, which suggests a role for lipid asymmetry in membrane bending and vesicle budding ${ }^{47,48}$.

Additional membrane-bound lipid transporters include ATP-binding cassette transporters that primarily function as lipid exporters rather than as flippases (BOX 2), and the plasma membrane scramblase, which functions in an ATP-independent reaction to randomize the phospholipid distribution across the plasma membrane $e^{35}$. The nature of the scramblase remains poorly understood. Evidence suggests that the loss of plasma membrane asymmetry could be caused by the action of one of the phospholipid scramblase family members ${ }^{53}$, a dysfunction of the aminophospholipid transporters ${ }^{54}$, or by the production of large quantities of non-bilayer-forming Cer by sphingomyelinases ${ }^{14}$.

\section{Selectivity in lipid transport}

All organelles contain at least some lipids that were synthesized elsewhere and obtained by transport. Lipid transport between organelles must be specific to maintain their unique lipid compositions. The plasma membrane, endosomes and lysosomes depend completely on lipid transport from other organelles that are actively engaged 
a Endoplasmic reticulum

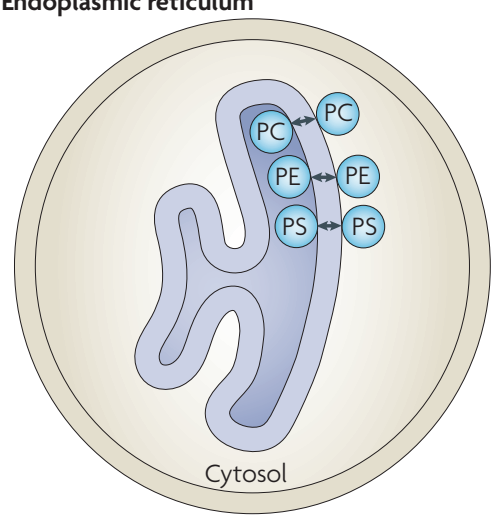

c Plasma membrane

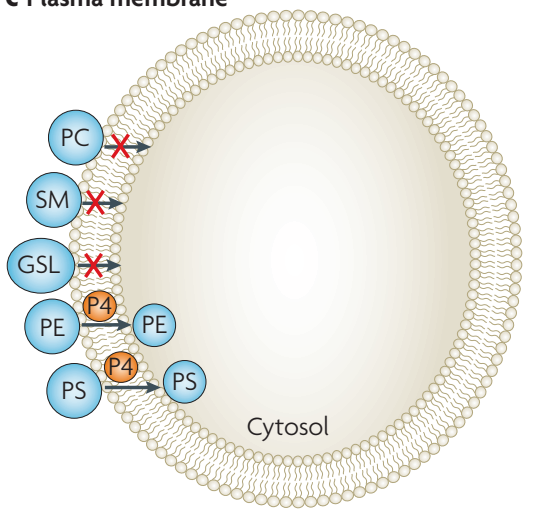

b Golgi

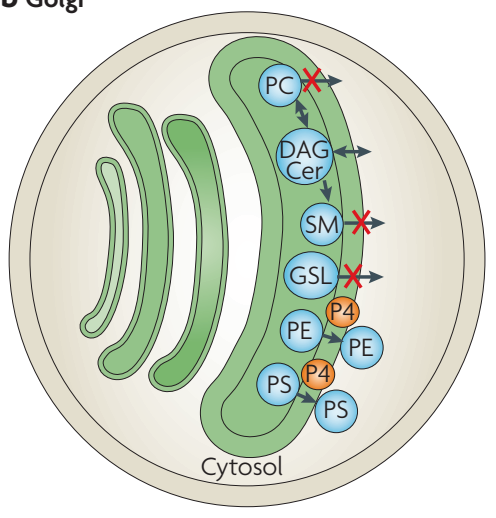

d Endosomes

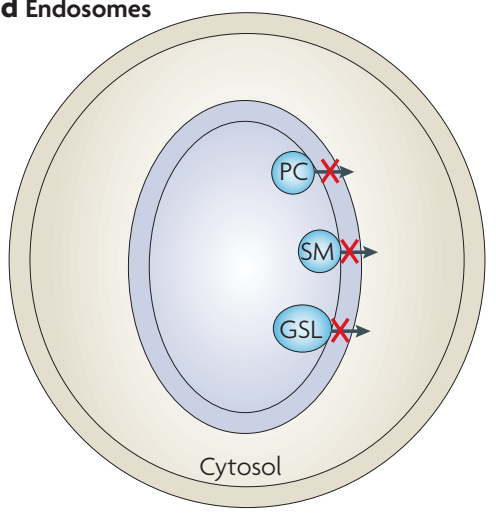

Figure 3 | Mechanisms for generating asymmetric lipid distribution. As lipids move from the endoplasmic reticulum (ER) to the Golgi, plasma membrane and into endosomes, intrinsic lipid transporters dictate the phospholipid distribution across the bilayer. a | In the ER, non-specific transbilayer equilibration of phospholipids has been demonstrated, and the membrane exhibits a nearly symmetric lipid distribution between bilayer leaflets. $\mathbf{b}$ | In the Golgi, P4 ATPases translocate phosphatidylserine (PtdSer; PS) and phosphatidylethanolamine (PtdEtn; PE) to the cytosolic face. Sphingomyelin (SM) is produced by SM synthase from ceramide (Cer) on the luminal side. Neither phosphatidylcholine (PtdCho; PC) nor SM molecules that are resident in the luminal leaflet are transported to the cytosolic face. Thus, asymmetry in the Golgi is generated by the specific transport of PtdSer and PtdEtn and lack of transport of SM and PtdCho. In SM synthesis, PtdCho is converted to diacylglycerol (DAG), which freely equilibrates across bilayers. DAG can serve as a substrate for the Golgi cholinephosphotransferase isozyme, the product of which is PtdCho. c|At the plasma membrane, P4 ATPases transport PtdSer andPtdEtn to the cytosolic face, with little or no transport of PtdCho or SM to the cytosolic face under basal conditions. This homeostatic distribution can be disrupted by activation of scramblase and/or inhibition of the P4 ATPases. $\mathbf{d}$ | Within endosomes, fluorescently labelled PtdCho and SM and glycosphingolipids (GSLs) were shown to be restricted to the luminal leaflet by a lack of specific transport mechanisms. P4 ATPases are recycled through endosomes ${ }^{123}$.

in synthesis. Lipid transport can occur by various mechanisms (FIG. 4a). First, lipids can laterally diffuse through membrane continuities, such as those that exist between the ER and the outer and inner nuclear membrane. Tubular connections have been observed between Golgi cisternae. In some instances, a diffusion barrier exists between otherwise continuous membranes, as is the case between apical and basolateral plasma membrane domains of epithelial cells. A barrier was observed exclusively in the exoplasmic leaflet of the plasma membrane, confirming that the GSLs that are enriched in the apical domain reside in this exoplasmic leaflet ${ }^{55}$. The major membrane transport pathway between cellular organelles in the secretory and endocytic pathways is through the budding and fusion of membrane vesicles. In contrast, organelles such as mitochondria and peroxisomes are not connected to the vesicular transport system and lipids are most likely to enter and leave these organelles as monomers, assisted by both soluble and membrane proteins.

Vesicular trafficking of membrane components. Secretory and endocytic organelles are connected by bidirectional vesicular transport. Following exit from the ER, the relatively free transbilayer movement of polar lipids becomes increasingly restricted. Several lipids become trapped in the exoplasmic leaflet of the bilayer and lose access to the cytosolic leaflet. The more complex sphingolipids (excluding GlcCer and GalCer) and PtdCho, which are all located in the exoplasmic leaflet of the bilayer, enter a pathway that requires vesicle-mediated transport. This is most evident from studies of the transport of SM and complex GSLs, which reveal the susceptibility to inhibition by metabolic poisons and genetic manipulations that disrupt vesicular transport between the Golgi and plasma membrane ${ }^{56}$. Only a small fraction of nascent sterol transport to the plasma membrane appears to be affected by inhibition of vesicular transport ${ }^{57}$.

Enrichment of sphingolipids and sterols along the secretory pathway predicts their preferential incorporation into anterograde vesicles or exclusion from retrograde vesicles. The situation is more complex in epithelial cells, in which separate transport pathways exist to the apical and basolateral plasma membrane. A general model has been proposed in which sphingolipids segregate from PtdCho in every compartment of the secretory pathway. In addition, in epithelial cells, GSLs should be preferentially included into apical precursor vesicles: the lipid raft hypothesis proposes that preferential interactions between lipids generate domains of specific lipid compositions to drive the sorting of membrane proteins ${ }^{8,58}$. The synthesis of GalCer in the ER of specialized cells ${ }^{17}$ and the recycling of newly synthesized GlcCer through the $\mathrm{ER}^{59}$ suggest that sphingolipids concentrate in rafts that already exist at the $\mathrm{ER}^{60}$.

The uptake of fluorescent sphingolipid and PtdCho analogues in mammalian cells proceeds by various endocytic pathways. The use of a fluorescent GSL with concentration-dependent changes in spectral properties revealed single endosomes with sphingolipids that were distributed in a non-random manner within the organelle, which is indicative of domains that are highly concentrated in sphingolipid. The LacCer analogue used was preferentially endocytosed by caveolae and was transported from the endosomes to the Golgi. This indicates that the endocytic recycling system uses lipid segregation mechanisms that are similar to those of the secretory pathway ${ }^{61}$. Extrapolation of these data to natural lipids is complicated by the effects of the fluorescent fatty acid on phase behaviour ${ }^{62}$. Interestingly, although the insertion of exogenous LacCer-stimulated caveolar endocytosis, an unnatural stereoisomer inhibited lipid clustering at the surface and blocked caveolar endocytosis of protein mark$\mathrm{ers}^{63}$. This suggests that lipid-lipid interactions regulate the initial step of the pathway. 


\section{Box 2 | ABC transporters: lipid exporters}

A rapidly expanding body of genetic data implicates ATP-binding cassette (ABC) transporters in the transbilayer movement of multiple lipids. The net reaction that is catalysed by ABC transporters is fundamentally different from that of P4 ATPases. The P4 ATPases allow lipids in the non-cytosolic membrane hemileaflet to move to the opposite cytosolic hemileaflet, whereas in almost all cases, the ABC transporters work in the opposite direction. Moreover, they expel a given lipid out of the membrane ${ }^{116}$. For most of the mammalian $A B C$ transporters that are involved in lipid transport, specific diseases or pathologies have been deduced by genetic means, but the mechanisms of action - and in many cases the substrates for the transporters - remain disputed. The main known $A B C$ transporters for lipids in humans are shown in the table below.

Whereas the multidrug transporters $A B C B 1$ and $A B C C 1$ translocate and export glucosylceramide (GlcCer) with a short, fluorescent fatty acid ${ }^{116}$, they do not translocate natural $\mathrm{GlcCer}^{59}$. Two bacterial $\mathrm{ABC}$ transporters that are of current importance to the fundamental questions of transbilayer and interbilayer lipid movement are MsbA and LolCDE. MsbA is involved in the translocation of immature lipopolysaccharide from the cytoplasmic to the exoplasmic side of the inner membrane of Gram-negative bacteria, and is coupled to lipid export from the inner to the outer membrane by periplasmic proteins. LolCDE detaches outer-membrane-specific lipoproteins from the exoplasmic face of the inner membrane onto a periplasmic receptor, an action that resembles the extrusion of dietary sitosterol by ABCG5/G8.

\begin{tabular}{|c|c|c|c|}
\hline Transporter & $\begin{array}{l}\text { Presumed } \\
\text { substrate }\end{array}$ & Location & $\begin{array}{l}\text { Disease when } \\
\text { inactivated }\end{array}$ \\
\hline ABCA1 & $\begin{array}{l}\text { Cholesterol, } \\
\text { PtdCho, PtdSer }\end{array}$ & Plasma membrane & $\begin{array}{l}\text { Tangier and familial } \\
\text { hypoalphalipoproteinaemia }\end{array}$ \\
\hline $\mathrm{ABCA} 3$ & $\begin{array}{l}\text { PtdCho, } \\
\text { cholesterol }\end{array}$ & $\begin{array}{l}\text { Lung lamellar } \\
\text { bodies }\end{array}$ & $\begin{array}{l}\text { Pulmonary surfactant } \\
\text { insufficiency }\end{array}$ \\
\hline ABCA4 & $\begin{array}{l}\text { N-retinylidene- } \\
\text { PtdEtn }\end{array}$ & Photoreceptor disks & $\begin{array}{l}\text { Stargardt and age-related } \\
\text { macular degeneration }\end{array}$ \\
\hline ABCA12 & GlcCer & Skin lamellar bodies & Harlequin ichthyosis \\
\hline ABCB4 & $\begin{array}{l}\text { PtdCho, } \\
\text { cholesterol }\end{array}$ & $\begin{array}{l}\text { Bile canalicular } \\
\text { membrane }\end{array}$ & Hepatic cholestasis \\
\hline $\mathrm{ABCD} 1$ & $\begin{array}{l}\text { Very long chain } \\
\text { fatty acyl-CoA }\end{array}$ & Peroxisomes & $\begin{array}{l}\text { X-linked } \\
\text { adrenoleukodystrophy }\end{array}$ \\
\hline ABCG1 & $\begin{array}{l}\text { PtdCho, SM, } \\
\text { cholesterol }\end{array}$ & Plasma membrane & Pulmonary lipidoses \\
\hline ABCG5/G8 & $\begin{array}{l}\text { Sitosterol, } \\
\text { cholesterol }\end{array}$ & Apical membrane & $\begin{array}{l}\text { Sitosterolaemia and } \\
\text { hypercholesterolaemia }\end{array}$ \\
\hline
\end{tabular}

ABC, ATP-binding cassette; GlcCer, glucosylceramide; PtdCho, phosphatidylcholine; PtdEtn, phosphatidylethanolamine; PtdSer, phosphatidylserine; SM, sphingomyelin.

Recruitment of cytosolic proteins. In many cases, arresting vesicle traffic using metabolic poisons or genetic mutations does not affect the transport of nascent glycerophospholipids and cholesterol between membranes ${ }^{22}$. Vesicle-independent movement has been demonstrated for PtdCho, PtdEtn and PtdSer between the ER, mitochondria, Golgi and the plasma membrane ${ }^{57,64-69}$; for cholesterol between the ER, lipid droplets, the plasma membrane and mitochondria of adrenal tissue ${ }^{57,70}$; for Cer from the ER to Golgi ${ }^{71}$; and finally, for GlcCer from Golgi to the ER and plasma membrane $\mathrm{e}^{59,72}$.

Genetic and biochemical experiments have implicated both protein and lipid assemblies on donor and acceptor membranes as important recognition components for membrane-membrane interactions that facilitate the non-vesicular lipid transfer processes. The lipid components include PtdIns4P, PA and PtdSer ${ }^{59,71,73-76}$, and the protein components include various structural gene products (CERT, FAPP2, VAP, $\underline{\mathrm{Pdr} 17}, \underline{\mathrm{Psd} 2}, \underline{\mathrm{StAR}}$,
TSPO, ORP $\left.{ }^{59,69-71,74-76}\right)$. They harbour canonical lipid transfer protein domains (such as START and GLTP) and domains that identify donor and acceptor membranes (C2, PH and FFAT), as well as ubiquitylation signals. A general model is emerging in which donor and acceptor membranes form transient zones of apposition and contact (FIG. 4). Their formation may depend on (or induce) the lateral segregation of lipids into domains. The contact sites could form restricted regions of lipid and protein diffusion within a given bilayer, and regions of transport protein diffusion between donor and acceptor membranes. The lipid and protein binding domains act to integrate the process as coincidence sensors that require the correct ligand for transport, the correct protein ligands on donor and/or acceptor membranes, and the correct organelle-identifying marker lipids (such as polyphosphoinositides) on donor and/or acceptor membranes. This combinatorial recognition process has the intrinsic ability to provide spatial and temporal cues that constitute a 'chemical roadmap' for vectorial lipid transport (FIG. 4b). Similar assemblies have been proposed for intermembrane lipid transport in plant chloroplasts ${ }^{77}$ and lipopolysaccharide transport in Gram-negative bacteria ${ }^{78}$, which both use multipartite ABC transporters.

Cytosolic proteins are also recruited to membranes to regulate dynamic aspects of lipid metabolism that must be integrated with vesicular protein traffic. The lipid transfer proteins $\underline{\operatorname{Sec} 14}$ in yeast and NIR2 in mammals sense membrane PtdIns and PtdCho content, and regulate the local synthesis of PtdCho at the cytosolic surface of the Golgi ${ }^{79,80}$. Suppression of Golgi PtdCho synthesis reduces the local consumption of DAG. This DAG pool is generated primarily as a product of sphingolipid synthesis in both yeast and mammalian cells, but may also arise from phospholipase reactions. The Golgi DAG content is thought to be crucial to both fission and fusion events within the organelle ${ }^{81,82}$.

\section{The behaviour of lipid membranes}

Membrane lipids can occur in various phases depending on their structure and environment (BOX 1). These phases have specific properties that determine the orientation and mobility of membrane lipids and proteins, and will therefore affect membrane functionality.

Lipid phase behaviour in biomembranes. Complications in studying lipid phase behaviours of biomembranes are the large size of the lipidome and the presence of membrane proteins at an approximately equal mass to lipids ${ }^{83,84}$. Each organellar membrane, each bilayer leaflet and each physically segregated domain within a leaflet contains only a subset of the lipidome. The identification of these domains and the elucidation of the relationships between physical state and function define the current limits of our understanding. The best data exist for the human red blood cell, in which the outer membrane leaflet is enriched in SM and PtdCho whereas the inner leaflet is enriched in PtdSer, PtdEtn and PtdIns. Even here, the cholesterol composition of each leaflet - a key component for phase behaviour - has proved indeterminate ${ }^{34}$. The in vivo asymmetry has been neglected in studies on model membranes that are 


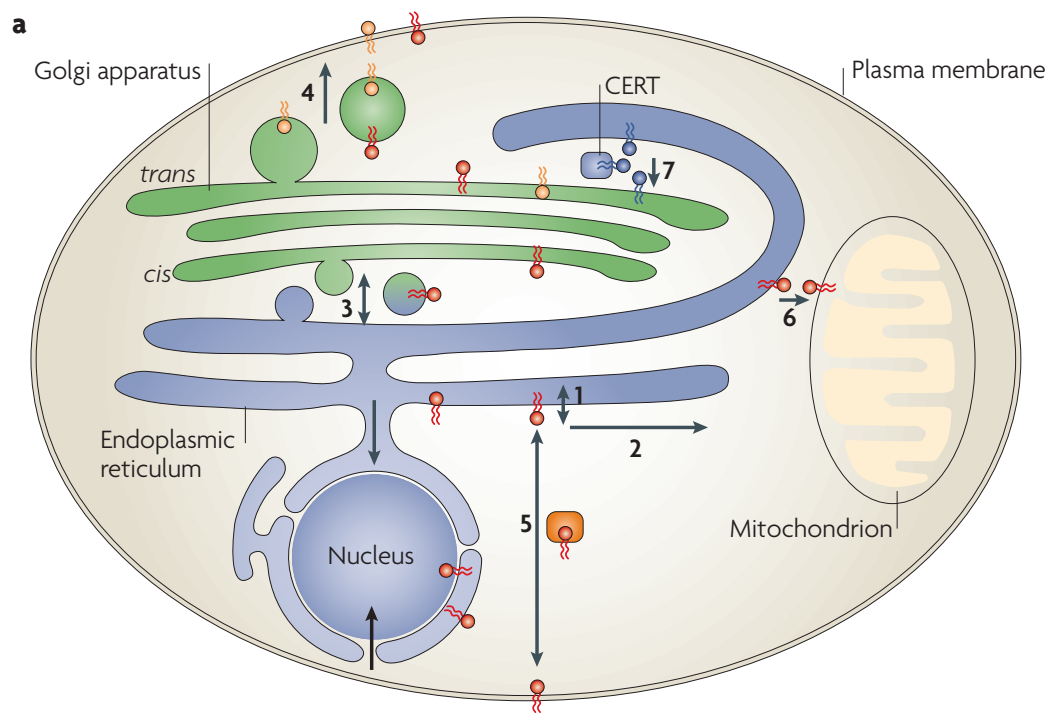

b

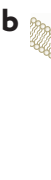

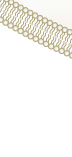
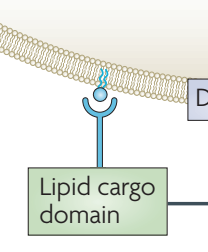

Donor

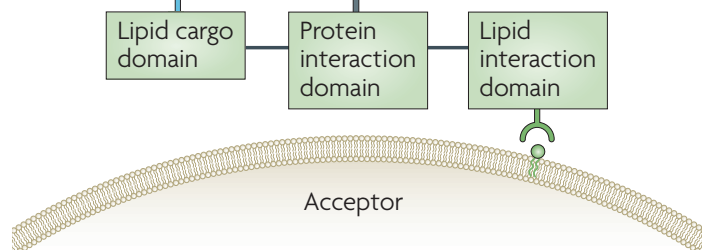

Figure 4 | Emerging models for lipid transport. a | Phosphatidylcholine (PtdCho; red) is synthesized on the cytosolic surface of the endoplasmic reticulum (ER; 1 ) and freely flips across the ER membrane. On both sides it diffuses laterally in any membrane (2). PtdCho travels through vesicles to the Golgi (3), the plasma membrane (4) and endosomes (not shown). It is transported by transfer proteins between the cytosolic surfaces of organelles (5), maybe through contact sites (6 and $\mathbf{7})$. The ceramide (Cer) transfer protein CERT transports Cer from the ER to the Golgi (7) for luminal sphingomyelin (SM) synthesis. SM cannot flip and travels in the luminal leaflet of the vesicle (4). Plasma-membrane enrichment of SM and cholesterol predicts their concentration at anterograde budding sites. The lipid symbols indicate the transmembrane orientation; the tails are buried in the bilayer interior. Adapted from REF. 8. b |A model for non-vesicular lipid transport predicts carrier protein interaction with a donor membrane protein, promoting ligation of the cargo lipid (blue). Cargo engagement facilitates carrier dissociation from the donor and diffusion to the acceptor membrane, which is recognized by distinct phosphoinositides (green). Binding between the carrier and the acceptor membrane induces cargo release, which is predicted to reduce the affinity of the carrier for the acceptor membrane and enable re-initiation of the cycle. Accessory proteins on donor and/or acceptor membranes may enhance the association and dissociation of carrier proteins. They may also facilitate the formation of contact sites, which restricts the diffusion path of the lipid carrier. The model provides the necessary elements of directionality and specificity to produce net lipid transfer.

\section{Caveolin}

The coat protein of caveolae.

It is anchored by a hydrophobic loop and 1-3 palmitoylated cysteines. Caveolin interacts with cholesterol and oligomerizes. composed of isolated plasma membrane lipids ${ }^{85}$. Perhaps progress can be made by organizing data and designing experiments following the successful approach used by geologists: thousands of different chemical components occur in all of the rocks on Earth, yet any given mineral has a far smaller, albeit often multicomponent, composition. This approach groups chemically similar components and maps the phase behaviour of typical examples of three or four of the groupings that describe a particular mineral ${ }^{86}$. By analogy, an appropriate system for phase behaviour studies in membranes would be a subset of the lipidome, over some fraction of the surface of one leaflet of a particular biomembrane. The molecular interactions that control the phase behaviour may be dominated by lipid-lipid forces (or in other cases by lipid-protein forces), coupling to the other leaflet and electrostatic binding of proteins or ions. These interactions can be dramatically affected by the activity of a membrane lipase or kinase that generates phase-changing products, and by crosslinking of lipids and/or proteins. The membrane phases cannot be at equilibrium, but instead exist at a steady state, with quasi-equilibrium describing the local patches of membrane in which component compositions persist for times that are longer than a time of interest, such as the time taken for a virus to bind or bud, or for a vesicle to fuse or bud, or for an enzyme to turnover.

Lipid mixtures that are similar to those of the ER membrane are expected to assume a homogeneous liquiddisordered phase at physiological temperatures. However, lipid mixtures that mimic the outer leaflet of the plasma membrane display a complex phase behaviour, with liquid-ordered $\left(l_{o}\right)$ and -disordered $\left(l_{d}\right)$ domains coexisting in the plane of the bilayer (BOX 3), while phase separation in the cytosolic leaflet may be induced by the outer leaflet organization (BOX 1). A similar situation is expected in the luminal leaflets of the trans-Golgi network and endosomes; although most of the actual evidence for the occurrence of lipid domains concerns the plasma membrane ${ }^{87}$ and the endosomes ${ }^{61}$, the necessity for lipid domains as an explanation for sphingolipid sorting in the Golgi was the original eye-opener for the field ${ }^{58}$.

Comparison of chemically defined models with cellular membranes reveals distinct, coexisting phases that can be observed by fluorescence microscopy of model membranes but not in the biomembranes of living cells ${ }^{88,89}$. The domains might exist but could be too small to see, being smaller than the wavelength of light $(<300 \mathrm{~nm})^{90}$. They might be confined to such small sizes by abundant membrane proteins or as a result of binding and stabilization by soluble proteins. Alternatively, such small domains might not normally be present, but instead might be more correctly described as the non-random mixing of dissimilar molecules within a single phase, characterized by clusters of up to 100 molecules, which appear and disappear on timescales that are controlled by diffusion. It is not clear if $l_{\mathrm{o}}$ - which does not show up in fluorescence microscopy images - is actually a separate phase from $l_{d}$; it might be a liquid phase that is highly non-ideal, with large clusters but not with separated phases. In either case, large-scale phase separation could be induced by relatively small changes in the relevant parameters, in the one case by the coalescence of small domains, in the other case by the stabilization and coalescence of the transient clusters. In cell membranes, coalescence is apparently induced by the sheer quantity of glycolipids and low temperature ${ }^{91}$, as well as during signalling ${ }^{92}$ (such as when Cer is produced by sphingomyelinases ${ }^{93}$ ), by membrane curving during budding ${ }^{94}$ or by lipid-ordering proteins such as caveolin ${ }^{87}$. These types of phase separation would require that the resting state of the membrane is close to a phase transition. 


\section{Box 3 | Phase behaviour of artificial membranes}

Different eukaryotic cellular membranes are likely to exhibit different phase behaviours. The figure shows three phase diagrams of three-component lipid mixtures (that is, mixtures that contain three different types of lipid). The large triangle describes the bilayer phase types that exist at all possible combinations of the three components, with any point within the triangle representing one defined lipid composition. The phases can be identified by techniques such as X-ray diffraction, fluorescence microscopy and various other spectroscopies to yield the complete diagram ${ }^{3,90,117-120}$. These multicomponent mixtures range from all lipids that are well-mixed in a single liquid phase over all compositions (type 1, top panel), to rich behaviour with multiple, different, compositionally distinct regions having two and three coexisting immiscible phases (type 3 , bottom panel). To model the outer leaflet of animal cell membranes, many researchers have chosen three-component mixtures containing cholesterol (CHOL), a high-melting phosphatidylcholine (PtdCho) or sphingomyelin, and a synthetic but naturally abundant PtdCho. For mono-unsaturated oleoyl in the sn2 position, these mixtures have \{solid + liquid\} coexistence at lower cholesterol concentrations ${ }^{3}$. However, the phase behaviour at higher cholesterol concentrations is not at all clear: whereas images of giant unilamellar vesicles show uniformity that is consistent with a single phase, various spectroscopy results are best interpreted by several regions of coexisting phases ${ }^{3,90}$. A unifying idea is that phases do coexist but are 'nanoscopic', having dimension(s) that are smaller than the wavelength of light, that is, $<300 \mathrm{~nm}^{90}$. Other studies have chosen the low-melting lipid for especially poor interaction with both the high-melting lipid and cholestero $^{117,118,120}$. Examples are dioleoyl-PtdCho (DOPC) and diphytanoyl-PtdCho carrying two unsaturated and two methylated fatty acyl chains, respectively. Here, the phase behaviour is complex, including at least one compositional region with two immiscible liquids, and in some mixtures, even a region of coexisting solid together with two immiscible liquids. Aside from DOPC and diphytanoyl-PtdCho, which are rare in biomembranes, a polyunsaturated fatty acid at sn2 of glycerophospholipids might itself drive phase separations, although full phase diagrams have not been determined ${ }^{119,121}$. DLPC (dilauroyl-PtdCho), DPPC (dipalmitoyl-PtdCho) and DSPC (distearoyl-PtdCho) are di-saturated PtdCho lipids with C12, C16 and C18 acyl chains, respectively.

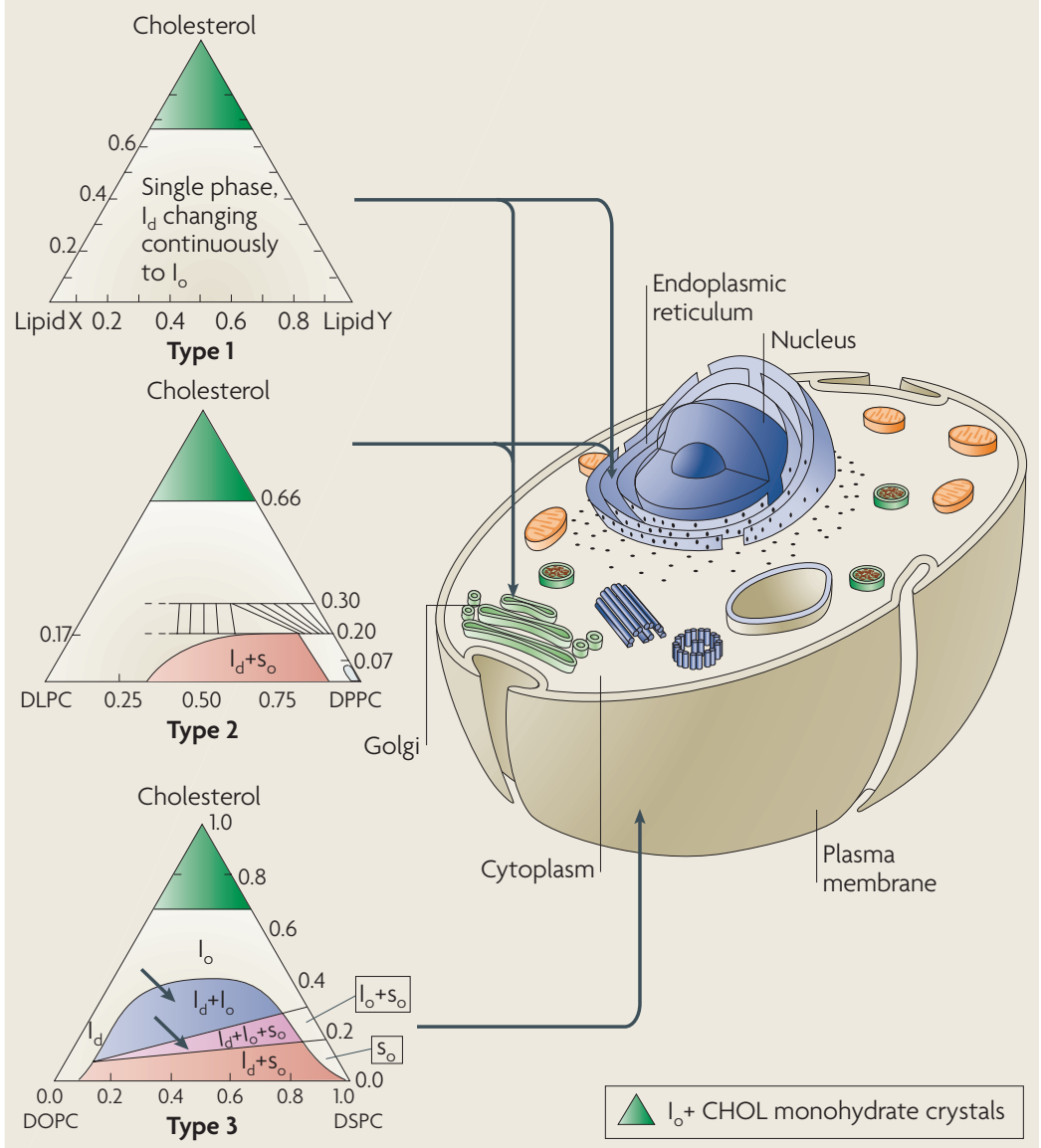

$\mathrm{I}_{\mathrm{d}}$, liquid-disordered; $\mathrm{l}_{\mathrm{o}}$, liquid-ordered; s, solid gel.
Mixture types that mimic the outer leaflet of the plasma membrane (BOX 3) have been examined in the presence of the lipolysis products Cer, DAG and LPC (or LPE) plus fatty acid. In bilayers that contain long-chain Cer and DAG molecules, a Cer- or DAG-rich solid phase can separate from the other lipids in the mixture ${ }^{95,96}$. Hexagonal and cubic phases have been found to form in simple binary mixtures containing DAG or Cer, but whether these phases actually form in more complex mixtures or in biomembranes (such as in mitochondria during apoptosis ${ }^{97}$ ) is not established. Lysophospholipids and fatty acids can be miscible with other components in lipid bilayers, but can also induce hexagonal and cubic phases. It is unclear whether these lipolysis products can actually build up to a sufficient concentration to form a new phase before their diffusion away from the site of synthesis, either in the plane of the membrane (which may be corralled in some way ${ }^{88,98}$ ) or out of the membrane.

Lipid phase boundaries. Phase separations within a planar bilayer induce surface domains of different lipid composition and phase properties. As shown in BOX 3, crossing a phase boundary could induce a change between one phase or two coexisting phases, or otherwise between two or three coexisting phases (arrows). As with allosteric enzymes, which enable cells to gain control of metabolic activity through the abrupt, cooperative behaviour of enzyme subunits, cells also profit from the cooperative behaviour of membranes. Phase separation creates a compositional phase boundary with a unique status: the phase boundaries are the physical sites at which the membrane properties change abruptly. Cells can use the sharp differences in membrane properties to colocalize some components and to separate others. Because phase separations depend on lipid composition, on binding of ions and molecules to the membrane, and on the aggregation state of membrane components, small changes in these parameters can control the phase behaviour. For example, phase separations may be driven by the loss of certain lipid components together with the addition of new ones, as occurs due to the activity of lipid kinases, phosphatases and hydrolases during signalling reactions, or by changes in $\mathrm{Ca}^{2+}$ concentration, or by the aggregation of immune receptors $^{99}$. As a complication to such behaviours, a cooperative transition during a membrane phase change can either be fast, trapping some components in non-equilibrium mixtures, or extremely slow, such as for hexagonal bilayer transitions.

Proteins and membrane behaviour. No relatively complete phase diagrams have been reported for proteins or peptides in multicomponent lipid mixtures. Helical peptides, including gramicidin $\mathrm{A}$, induce non-bilayer phases at concentrations of a few mole percent ${ }^{100}$. Most simple helical peptides induce a non-bilayer phase at low mole fractions ${ }^{101}$. Such studies highlight the weakness of extrapolating from simple model peptides in lipid bilayers to biomembranes, which have far higher protein concentrations ${ }^{84,102}$. The induction of non-bilayer phases by peptides at rather low concentrations shows that 
pore formation, biomembrane curvature, and perhaps vesiculation and tubulation, may be under the control of proteins that reside in or bind to biomembranes.

Proteins can show a clear preference for a particular phase. For coexisting liquid and solid phases in model bilayers, a peptide or protein that is anchored to the membrane by an $\alpha$-helix typically prefers the liquid phase. Partitioning into the solid phase in preference to the liquid phase only occurs for precise hydrophobic matching with the solid. For coexisting $l_{o}$ and $l_{d}$ domains, most membrane-bound peptides partition out of $l_{0}$ and into $1_{d}{ }^{103,104}$. Exceptions are peptides of the caveolin-1 scaffolding domain and at least some glycosylphosphatidylinositol (GPI)-anchored proteins ${ }^{105}$. Cholesterol binding domains also seem to exist that have high affinity for the cholesterol-rich compositional regions of a bilayer ${ }^{105}$. However, it is not yet clear if the binding is to pre-existing cholesterol-rich domains, or to what extent this binding occurs, or if the protein induces a distinct domain to form. Important questions remain about whether and how lipid rafts in vesicular transport recruit specific proteins, such as the SNAREs (soluble $\mathrm{N}$-ethylmaleimide-sensitive factor attachment protein receptors) that are required for targeting and docking these vesicles, and whether such proteins may actually accumulate at domain boundaries ${ }^{106}$.

A related question regarding the function of various lipids is whether lipids that are in contact with membrane proteins form a shell with special properties; for example, a tendency to nucleate membrane rafts ${ }^{102}$. In several well-studied cases, proteins that were reconstituted into lipid bilayers show surprisingly little binding preference for a shell of particular lipids based on lipid chain length or headgroup ${ }^{107}$. The enzymatic and transport activities of membrane-bound reconstituted proteins show little preference for a particular lipid chain length over a broad range, as in the case of the $\mathrm{Ca}^{2+}$-ATPase ${ }^{108}$. For $\mathrm{Na}^{+} / \mathrm{K}^{+}$ATPase, only CL shows several-fold binding affinity compared with other phospholipids ${ }^{109}$ but CL resides in a different cellular membrane. By contrast, for bovine rhodopsin, Gawrisch and colleagues found that a model of specific lipid binding (either via the headgroup or via unsaturation) was needed to explain data that showed that the binding affinity of PtdCho was approximately equal to PtdEtn, which has a greater binding affinity than PtdSer, and the binding affinity of PUFA (polyunsaturated fatty acid) was greater than that of monounsaturated fatty acid $^{110}$. Andersen and Koeppe have suggested a view of lipid binding to membrane proteins that emphasizes the overall energetics of the interactions, rather than the affinity of specific lipids for a protein ${ }^{111}$. An advantage of this view is that it may apply equally well to protein behaviour within single or multiple membrane phases. This complements the notions that differential interactions between the various membrane components result in small but definite heterogeneities in the membrane $e^{92,112}$, which can be termed lipid rafts if they are membrane rafts that depend on lipid-lipid interactions for their existence.

\section{Conclusions and future directions}

Eukaryotic cells have a tremendous array of lipid metabolizing enzymes with unique distributions over their various membranes. However, these do not explain all of the steady-state lipid compositions. Specificity in lipid transport is also required. On the one hand, ever more proteins are discovered that are involved in monomeric lipid transport and impose specificity and directionality. On the other hand, vesicular transport uses lipid phase behaviour to laterally segregate lipids and proteins and include specific subsets in budding vesicles. Similar segregations are induced in numerous signal transduction pathways. In both cases, phosphoinositide dynamics have a regulatory function through the recruitment of cytosolic proteins. Despite impressive progress in the biophysics of model systems, the application of these approaches to cellular systems has been hampered by the stunning complexity of the cellular lipidome. Although studies of membranes can now benefit from the large-scale detailed analyses of lipid molecular species, there is currently a paucity of data regarding key points, including: complete lipid compositional analysis for each organelle, the lipid compositions of each bilayer leaflet, the coupling of phase behaviours of the two leaflets, and the influence of proteins on lipid phase behaviours. To overcome the technical and conceptual barriers confronted by the field, a multidisciplinary approach is required, involving biochemists, cell biologists, physicists and information technologists working together. Such progress will highlight the basic working principles of cells and tissues, but will also allow fundamental insights into the pathogenicity of disease. Important human diseases, such as atherosclerosis, infectious diseases, Alzheimer's disease and cancer, all have a lipid component in their epidemiology. A molecular understanding of the contribution of lipids to the disease process will allow the development of novel approaches to prevention, diagnosis and cure.
1. Sud, M. et al. LMSD: LIPID MAPS structure database. Nucleic Acids Res. 35, D527-D532 (2007).

2. Feigenson, G. W. Phase behavior of lipid mixtures Nature Chem. Biol. 2, 560-563 (2006)

Feigenson, G. W. Phase boundaries and biological membranes. Annu. Rev. Biophys. Biomol. Struct. 36, 63-77 (2007)

4. Marsh, M. \& Helenius, A. Virus entry: open sesame. Cell 124, 729-740 (2006).

Cell biology studies, live-cell imaging and systems biology show that many of the multiple and subtly different pathways that animal viruses use to enter host cells require specific lipids.

5. van Meer, G. Cellular lipidomics. EMBO J. 24, 3159-3165 (2005).
6. Marsh, D. Lateral pressure profile, spontaneous curvature frustration, and the incorporation and conformation of proteins in membranes. Biophys. J. 93 3884-3899 (2007)

7. Dowhan, W. \& Bogdanov, M. in Biochemistry of Lipids, Lipoproteins and Membranes Vol. 36 (eds Vance, D. E. \& Vance, J. E.) 1-35 (Elsevier, Amsterdam, 2002).

8. van Meer, G. \& Lisman, Q. Sphingolipid transport: rafts and translocators. J. Biol. Chem. 277, 25855-25858 (2002).

9. Huang, J. \& Feigenson, G. W. A microscopic interaction model of maximum solubility of cholesterol in lipid bilayers. Biophys. J. 76, 2142-2157 (1999). The interactions of cholesterol with membrane lipid lead to abrupt jumps in cholesterol chemical potential because of the hydrophobic interaction, which forces phospholipid headgroups to shield cholesterol from water, as described here by the umbrella model.

10. Ali, M. R., Cheng, K. H. \& Huang, J. Ceramide drives cholesterol out of the ordered lipid bilayer phase into the crystal phase in 1-palmitoyl-2-oleoyl-sn-glycero-3phosphocholine/cholesterol/ceramide ternary mixtures. Biochemistry 45, 12629-12638 (2006).

11. Meyer zu Heringdorf, D. \& Jakobs, K. H. Lysophospholipid receptors: signalling, pharmacology and regulation by lysophospholipid metabolism. Biochim. Biophys. Acta 1768, 923-940 (2007).

12. Fernandis, A. Z. $\&$ Wenk, M. R. Membrane lipids as signaling molecules. Curr. Opin. Lipidol. 18, 121-128 (2007).

13. Kolesnick, R. \& Hannun, Y. A. Ceramide and apoptosis Trends Biochem. Sci. 24, 224-225 (1999). 
14. Tepper, A. D. et al. Sphingomyelin hydrolysis to ceramide during the execution phase of apoptosis results from phospholipid scrambling and alters cell-surface morphology. J. Cell Biol. 150, 155-164 (2000).

15. Megha, Sawatzki, P., Kolter, T., Bittman, R. \& London, E. Effect of ceramide $N$-acyl chain and polar headgroup structure on the properties of ordered lipid domains (lipid rafts). Biochim. Biophys. Acta 1768 2205-2212 (2007).

16. Bell, R. M., Ballas, L. M. \& Coleman, R. A. Lipid topogenesis. J. Lipid Res. 22, 391-403 (1981)

7. Sprong, H. et al. UDP-galactose:ceramide galactosyltransferase is a class I integral membrane protein of the endoplasmic reticulum. J. Biol. Chem. 273, 25880-25888 (1998)

18. Rusinol, A. E., Cui, Z., Chen, M. H. \& Vance, J. E. A unique mitochondria-associated membrane fraction from rat liver has a high capacity for lipid synthesis and contains pre-Golgi secretory proteins including nascent lipoproteins. J. Biol. Chem. 269,

27494-27502 (1994)

Provides evidence for stable physical associations between the ER and mitochondria, and also identifies biochemical properties of this compartment that are distinct from the individual organelles.

19. Pichler, H. et al. A subfraction of the yeast endoplasmic reticulum associates with the plasma membrane and has a high capacity to synthesize lipids. Eur. J. Biochem. 268, 2351-2361 (2001)

20. Futerman, A. H. \& Riezman, H. The ins and outs of sphingolipid synthesis. Trends Cell Biol. 15, 312-318 (2005).

21. Henneberry, A. L., Wright, M. M. \& McMaster, C. R. The major sites of cellular phospholipid synthesis and molecular determinants of fatty acid and lipid head group specificity. Mol. Biol. Cell 13, 3148-3161 (2002).

22. Voelker, D. R. Bridging gaps in phospholipid transport. Trends Biochem. Sci. 30, 396-404 (2005). Summarizes biochemical and genetic elements of non-vesicular phospholipid transport with highlighted emphasis on PtdSer transport processes in yeast.

23. Di Paolo, G. $\&$ De Camilli, P. Phosphoinositides in cell regulation and membrane dynamics. Nature 443 651-657 (2006)

24. Geta Tafesse, F. et al. Both sphingomyelin synthases SMS1 and SMS2 are required for sphingomyelin homeostasis and growth in human HeLa cells. J. Biol. Chem. 282, 17537-17547 (2007)

25. Li, Z. et al. Inhibition of sphingomyelin synthase (SMS) affects intracellular sphingomyelin accumulation and plasma membrane lipid organization. Biochim. Biophys. Acta 1771, 1186-1194 (2007).

26. Kobayashi, T. et al. Separation and characterization of late endosomal membrane domains. J. Biol. Chem. 277, 32157-32164 (2002)

27. Matsuo, $\mathrm{H}$ et al. Role of LBPA and Alix in multivesicular liposome formation and endosome organization. Science 303, 531-534 (2004).

28. Kolter, T. \& Sandhoff, K. Principles of lysosomal membrane digestion: stimulation of sphingolipid degradation by sphingolipid activator proteins and anionic lysosomal lipids. Annu. Rev. Cell Dev. Biol. 21 81-103 (2005).

29. Vance, D. E. \& Vance, J. E. Biochemistry of Lipids, Lipoproteins and Membranes (Elsevier, Amsterdam 2002)

30. Nagle, C. A. et al. Hepatic overexpression of glycerol$s n-3$-phosphate acyltransferase 1 in rats causes insulin resistance. J. Biol. Chem. 282, 14807-14815 (2007).

31. Choi, J. Y., Wu, W. I. \& Voelker, D. R. Phosphatidylserine decarboxylases as genetic and biochemical tools for studying phospholipid traffic. Anal. Biochem. 347, 165-175 (2005).

32. Daum, G. Lipids of mitochondria. Biochim. Biophys. Acta 822, 1-42 (1985)

33. Strauss, J. F., Kishida, T., Christenson, L. K., Fujimoto, T. $\&$ Hiroi, H. START domain proteins and the intracellular trafficking of cholesterol in steroidogenic cells. Mol. Cell. Endocrinol. 202, 59-65 (2003).

34. Devaux, P. F. \& Morris, R. Transmembrane asymmetry and lateral domains in biological membranes. Traffic 5, 241-246 (2004)

35. Daleke, D. L. Phospholipid flippases. J. Biol. Chem. 282, 821-825 (2007)

36. Pomorski, T. \& Menon, A. K. Lipid flippases and their biological functions. Cell. Mol. Life Sci. 63 2908-2921 (2006).
37. Anglin, T. C., Liu, J. \& Conboy, J. C. Facile lipid flip-flop in a phospholipid bilayer induced by gramicidin $\mathrm{A}$ measured by sum-frequency vibrational spectroscopy. Biophys. J. 92, L01-L03 (2007)

38. Papadopulos, A. et al. Flippase activity detected with unlabeled lipids by shape changes of giant unilamellar vesicles. J. Biol. Chem. 282, 15559-15568 (2007).

39. López-Montero, I. et al. Rapid transbilayer movement of ceramides in phospholipid vesicles and in human erythrocytes. J. Biol. Chem. 280, 25811-25819 (2005)

40. Ganong, B. R. \& Bell, R. M. Transmembrane movement of phosphatidylglycerol and diacylglycero sulfhydryl analogues. Biochemistry 23, 4977-4983 (1984).

41. Bai, J. \& Pagano, R. E. Measurement of spontaneous transfer and transbilayer movement of BODIPYlabeled lipids in lipid vesicles. Biochemistry 36, 8840-8848 (1997)

42. Steck, T. L., Ye, J. \& Lange, Y. Probing red cell membrane cholesterol movement with cyclodextrin. Biophys. J. 83, 2118-2125 (2002).

43. Cullis, P. R. et al. Influence of $\mathrm{pH}$ gradients on the transbilayer transport of drugs, lipids, peptides and metal ions into large unilamellar vesicles. Biochim. Biophys. Acta 1331, 187-211 (1997).

44. Kol, M. A., de Kroon, A. I., Killian, J. A. \& de Kruijff, B. Transbilayer movement of phospholipids in biogenic membranes. Biochemistry 43, 2673-2681 (2004). Summarizes the data and hypotheses that support a generic system for the non-selective transbilayer movement of lipids in the ER of eukaryotes and in the cytoplasmic membranes of bacteria.

45. Helenius, J. et al. Translocation of lipid-linked oligosaccharides across the ER membrane requires Rft 1 protein. Nature 415, 447-450 (2002)

46. Alaimo, C. et al. Two distinct but interchangeable mechanisms for flipping of lipid-linked oligosaccharides. EMBO J. 25, 967-976 (2006).

47. Graham, T. R. Flippases and vesicle-mediated protein transport. Trends Cell Biol. 14, 670-677 (2004). Summarizes important relationships between vesicular protein traffic and transbilayer phospholipid transport by P-type ATPases.

48. Pomorski, T. et al. Drs2p-related P-type ATPases Dnf $1 p$ and Dnf2p are required for phospholipid translocation across the yeast plasma membrane and serve a role in endocytosis. Mol. Biol. Cell 14 1240-1254 (2003).

An important paper defining the participation of plasma membrane P-type ATPases in yeast in the transbilayer movement of aminoglycerophospholipids and their interplay with endocytic processes.

49. Riekhof, W. R. \& Voelker, D. R. Uptake and utilization of lyso-phosphatidylethanolamine by Saccharomyces cerevisiae. J. Biol. Chem. 281, 36588-36596 (2006).

0. Riekhof, W. R. et al. Lyso-phosphatidylcholine metabolism in Saccharomyces cerevisiae. The role of P-type ATPases in transport and a broad specificity acyltransferase in acylation. J. Biol. Chem. 21 Oct 2007 (doi:10.1074/jbc.M706718200)

51. Natarajan, P., Wang, J., Hua, Z. \& Graham, T. R. Drs2p-coupled aminophospholipid translocase activity in yeast Golgi membranes and relationship to in vivo function. Proc. Natl. Acad. Sci. USA 101

10614-10619 (2004)

52. Alder-Baerens, N., Lisman, Q., Luong, L., Pomorski, T. $\&$ Holthuis, J. C. Loss of P4 ATPases Drs2p and Dnf3p disrupts aminophospholipid transport and asymmetry in yeast post-Golgi secretory vesicles. Mol. Biol. Cell 17, 1632-1642 (2006)

53. Wang, X. et al. C. elegans mitochondrial factor WAH-1 promotes phosphatidylserine externalization in apoptotic cells through phospholipid scramblase SCRM-1. Nature Cell Biol. 9, 541-549 (2007).

54. Züllig, S. et al. Aminophospholipid translocase TAT-1 promotes phosphatidylserine exposure during C. elegans apoptosis. Curr. Biol. 17, 994-999 (2007).

55. van Meer, G. \& Simons, K. The function of tight junctions in maintaining differences in lipid composition between the apical and the basolateral cell surface domains of MDCK cells. EMBO J. 5 1455-1464 (1986).

56. Young, W. W. Jr, Lutz, M. S. \& Blackburn, W. A. Endogenous glycosphingolipids move to the cell surface at a rate consistent with bulk flow estimates. J. Biol. Chem. 267, 12011-12015 (1992)

57. Baumann, N. A. et al. Transport of newly synthesized sterol to the sterol-enriched plasma membrane occurs via nonvesicular equilibration. Biochemistry 44 5816-5826 (2005)
58. Simons, K. \& van Meer, G. Lipid sorting in epithelial cells. Biochemistry 27, 6197-6202 (1988)

59. Halter, D. et al. Pre- and post-Golgi translocation of glucosylceramide in glycosphingolipid synthesis $\mathrm{J}$. Cell Biol. 179, 101-115 (2007).

Suggests that the late Golgi protein FAPP2 transports GlcCer that is destined for complex glycolipid synthesis back to the ER, whereas GlcCer translocation to the cell surface depends on a proton gradient

60. Smith, D. C. et al. The association of Shiga-like toxin with detergent-resistant membranes is modulated by glucosylceramide and is an essential requirement in the endoplasmic reticulum for a cytotoxic effect. Mol. Biol. Cell 17, 1375-1387 (2006).

61. Sharma, D. K. et al. Glycosphingolipids internalized via caveolar-related endocytosis rapidly merge with the clathrin pathway in early endosomes and form microdomains for recycling. J. Biol. Chem. 278 7564-7572 (2003)

Demonstrates the non-uniform distribution of fluorescent phospholipids within individual endosomes.

62. Wang, T. Y. \& Silvius, J. R. Different sphingolipids show differential partitioning into sphingolipid/cholesterolrich domains in lipid bilayers. Biophys. J. 79 1478-1489 (2000)

63. Singh, R. D. et al. Inhibition of caveolar uptake, SV40 infection, and $\beta 1$-integrin signaling by a nonnatural glycosphingolipid stereoisomer. J. Cell Biol. 176, 895-901 (2007)

64. Sleight, R. G. \& Pagano, R. E. Rapid appearance of newly synthesized phosphatidylethanolamine at the plasma membrane. J. Biol. Chem. 258, 9050-9058 (1983).

65. Kaplan, M. R. \& Simoni, R. D. Intracellular transport of phosphatidylcholine to the plasma membrane. J. Cell Biol. 101, 441-445 (1985).

66. Voelker, D. R. Characterization of phosphatidylserine synthesis and translocation in permeabilized animal cells. J. Biol. Chem. 265, 14340-14346 (1990).

67. Vance, J. E., Aasman, E. J. \& Szarka, R. Brefeldin A does not inhibit the movement of phosphatidylethanolamine from its sites of synthesis to the cell surface. J. Biol. Chem. 266, 8241-8247 (1991).

68. Wu, W. I. \& Voelker, D. R. Characterization of phosphatidylserine transport to the locus of phosphatidylserine decarboxylase 2 in permeabilized yeast. J. Biol. Chem. 276, 7114-7121 (2001).

69. Schumacher, M. M., Choi, J. Y. \& Voelker, D. R. Phosphatidylserine transport to the mitochondria is regulated by ubiquitination. J. Biol. Chem. 277 , 51033-51042 (2002)

70. Papadopoulos, V. et al. Translocator protein (18kDa): new nomenclature for the peripheral-type benzodiazepine receptor based on its structure and molecular function. Trends Pharmacol. Sci. 27 402-409 (2006)

Summarizes the major protein constituents involved in non-vesicular import of cholesterol into the mitochondria of cells involved in steroid hormone synthesis.

71. Hanada, K. et al. Molecular machinery for nonvesicular trafficking of ceramide. Nature $\mathbf{4 2 6}$ 803-809 (2003).

An important paper defining the genetic and biochemical mechanisms for non-vesicular transport of ceramide between the ER and the Golgi apparatus.

72. Warnock, D. E., Lutz, M. S., Blackburn, W. A., Young, W. W. Jr \& Baenziger, J. U. Transport of newly synthesized glucosylceramide to the plasma membrane by a non-Golgi pathway. Proc. Natl. Acad. Sci. USA 91, 2708-2712 (1994).

73. Trotter, P. J., Wu, W. I., Pedretti, J., Yates, R. \& Voelker, D. R. A genetic screen for aminophospholipid transport mutants identifies the phosphatidylinositol 4-kinase, STT4p, as an essential component in phosphatidylserine metabolism. J. Biol. Chem. 273. 13189-13196 (1998).

74. Wu, W. I. \& Voelker, D. R. Reconstitution of phosphatidylserine transport from chemically defined donor membranes to phosphatidylserine decarboxylase 2 implicates specific lipid domains in the process. J. Biol. Chem. 279, 6635-6642 (2004)

75. Raychaudhuri, S., Im, Y. J., Hurley, J. H. \& Prinz, W. A Nonvesicular sterol movement from plasma membrane to ER requires oxysterol-binding protein related proteins and phosphoinositides. J. Cell Biol. 173, 107-119 (2006). 
76. D'Angelo, G. et al. Glycosphingolipid synthesis requires FAPP2 transfer of glucosylceramide. Nature $\mathbf{4 4 9}$, 62-67 (2007)

Shows that FAPP2, a protein that is associated with the generation of transport carriers from the Golgi to the plasma membrane, appears to be a GlcCer transfer protein with a pivotal role in complex GSL synthesis.

77. Awai, K., Xu, C., Tamot, B. \& Benning, C. A phosphatidic acid-binding protein of the chloroplast inner envelope membrane involved in lipid trafficking. Proc. Natl Acad. Sci. USA 103, 10817-10822 (2006) Defines transport and intermembrane recognition components for moving phospholipids between the outer and inner chloroplast membranes.

78. Tefsen, B., Geurtsen, J., Beckers, F., Tommassen, J. \& de Cock, H. Lipopolysaccharide transport to the bacterial outer membrane in spheroplasts. J. Biol. Chem. 280, 4504-4509 (2005)

79. Mousley, C. J., Tyeryar, K. R., Vincent-Pope, P. \& Bankaitis, V. A. The Sec14-superfamily and the regulatory interface between phospholipid metabolism and membrane trafficking. Biochim. Biophys. Acto 1771, 727-736 (2007)

80. Litvak, V., Dahan, N., Ramachandran, S., Sabanay, H. \& Lev, S. Maintenance of the diacylglycerol level in the Golgi apparatus by the Nir2 protein is critical for Golgi secretory function. Nature Cell Biol. 7, 225-234 (2005).

81. Chernomordik, L., Kozlov, M. M. \& Zimmerberg, J. Lipids in biological membrane fusion. J. Membr. Biol. 146, 1-14 (1995).

82. Shemesh, T., Luini, A., Malhotra, V., Burger, K. N. \& Kozlov, M. M. Prefission constriction of Golgi tubular carriers driven by local lipid metabolism: a theoretical model. Biophys. J. 85, 3813-3827 (2003)

83. Gennis, R. B. Biomembranes. Molecular Structure and Function (Springer Verlag, New York, 1989).

84. Takamori, S. et al. Molecular anatomy of a trafficking organelle. Cell 127, 831-846 (2006). A careful reconstruction of synaptic vesicles shows that cholesterol and phospholipids (molar ratio 0.8 ) cover $\sim 70 \%$ of the surface while transmembrane helices cover $20 \%$, with a lipid/protein ratio of 0.75 (w/w).

85. Dietrich, C., Volovyk, Z. N., Levi, M., Thompson, N. L. \& Jacobson, K. Partitioning of Thy-1, GM1, and crosslinked phospholipid analogs into lipid rafts reconstituted in supported model membrane monolayers. Proc. Natl Acad. Sci. USA 98 10642-10647 (2001).

86. Morse, S. A. Basalts and Phase Diagrams (Springer Verlag, New York, 1980)

87. Parton, R. G. Ultrastructural localization of gangliosides: GM1 is concentrated in caveolae. J. Histochem. Cytochem. 42, 155-166 (1994)

88. Kusumi, A. et al. Paradigm shift of the plasma membrane concept from the two-dimensional continuum fluid to the partitioned fluid: high-speed single-molecule tracking of membrane molecules. Annu. Rev. Biophys. Biomol. Struct. 34, 351-378 (2005). Biological membrane lipids and proteins are divided into regions, some tens of nanometres in size, which have distinct molecular components and properties.

89 Lagerholm, B. C. Weinreb, G. E., Jacobson, K. \& Thompson, N. L. Detecting microdomains in intact cell membranes. Annu. Rev. Phys. Chem. 56, 309-336 (2005).

90. Feigenson, G. W. \& Buboltz, J. T. Ternary phase diagram of dipalmitoyl-PC/dilauroyl-PC/cholesterol: nanoscopic domain formation driven by cholesterol. Biophys. J. 80, 2775-2788 (2001).

91. Meder, D., Moreno, M. J., Verkade, P., Vaz, W. L. \& Simons, K. Phase coexistence and connectivity in the apical membrane of polarized epithelial cells. Proc. Natl Acad. Sci. USA 103, 329-334 (2006).

92. Kusumi, A., Koyama-Honda, I. \& Suzuki, K. Molecular dynamics and interactions for creation of stimulation-induced stabilized rafts from small unstable steady-state rafts. Traffic 5, 213-230 (2004).
93. Bollinger, C. R., Teichgraber, V. \& Gulbins, E. Ceramideenriched membrane domains. Biochim. Biophys. Acta 1746, 284-294 (2005).

94. Roux, A. et al. Role of curvature and phase transition in lipid sorting and fission of membrane tubules. EMBO J. 24, 1537-1545 (2005)

95. Chiantia, S., Kahya, N., Ries, J. \& Schwille, P. Effects of ceramide on liquid-ordered domains investigated by simultaneous AFM and FCS. Biophys. J. 90 4500-4508 (2006)

96. Sot, J., Bagatolli, L A., Goni, F M \& Alonso, A Detergent-resistant, ceramide-enriched domains in sphingomyelin/ceramide bilayers. Biophys. J. 90 903-914 (2006).

97. Anishkin, A., Sukharev, S. \& Colombini, M. Searching for the molecular arrangement of transmembrane ceramide channels. Biophys. J. 90, 2414-2426 (2006).

98. Dietrich, C., Yang, B., Fujiwara, T., Kusumi, A. \& Jacobson, K. Relationship of lipid rafts to transient confinement zones detected by single particle tracking. Biophys. J. 82, 274-284 (2002)

99. Sengupta, P., Baird, B. \& Holowka, D. Lipid rafts, fluid fluid phase separation, and their relevance to plasma membrane structure and function. Semin. Cell Dev. Biol. 18, 583-590 (2007)

100. Dibble, A. R. \& Feigenson, G. W. Detection of coexisting fluid phospholipid phases by equilibrium $\mathrm{Ca}^{2+}$ binding: peptide-poor $\mathrm{L} \alpha$ and peptide-rich HII phase coexistence in gramicidin $\mathrm{A}^{\prime} /$ phospholipid dispersions. Biochemistry 33, 12945-12953 (1994)

101. Lewis, R. N. et al. Studies of the minimum hydrophobicity of $\alpha$-helical peptides required to maintain a stable transmembrane association with phospholipid bilayer membranes. Biochemistry 46 1042-1054 (2007).

102. Jacobson, K., Mouritsen, O. G. \& Anderson, R. G. Lipid rafts: at a crossroad between cell biology and physics. Nature Cell Biol. 9, 7-14 (2007). Proposes a model whereby transmembrane helices cover $15 \%$ of the surface and physically contact $30 \%$ of the membrane lipids, termed shell lipids. On receiving a signal, proteins control phase behaviour by combining their shell with similar lipid shells of other proteins.

103. Shogomori, H. et al. Palmitoylation and intracellular domain interactions both contribute to raft targeting of linker for activation of T cells. J. Biol. Chem. $\mathbf{2 8 0}$, 18931-18942 (2005)

104. Brown, D. A. Lipid rafts, detergent-resistant membranes, and raft targeting signals. Physiology 21 , 430-439 (2006)

The use of detergent resistance to study the properties of biological membranes gives indirect information about protein and lipid phase preferences, but does not capture a snapshot of actual phase behaviour.

105. Epand, R. M. Cholesterol and the interaction of proteins with membrane domains. Prog. Lipid Res. 45, 279-294 (2006).

106. Hancock, J. F. Lipid rafts: contentious only from simplistic standpoints. Nature Rev. Mol. Cell Biol. 7 456-462 (2006).

A critical discussion of lipid rafts, stressing that protein-protein interactions make major contributions to the stability of lipid-based domains, and that proteins and specific lipids such as cholesterol may accumulate at and affect domain boundaries.

107. London, E. \& Feigenson, G. W. Fluorescence quenching in model membranes. 2. Determination of local lipid environment of the calcium adenosinetriphosphatase from sarcoplasmic reticulum. Biochemistry 20 1939-1948 (1981).

108. Caffrey, M \& Feigenson, G. W. Fluorescence quenching in model membranes. 3. Relationship between calcium adenosinetriphosphatase enzyme activity and the affinity of the protein for phosphatidylcholines with different acyl chain characteristics. Biochemistry 20, 1949-1961 (1981).
109. Esmann, M. \& Marsh, D. Lipid-protein interactions with the Na, K-ATPase. Chem. Phys. Lipids 141 94-104 (2006).

110. Soubias, O., Teague, W. E. \& Gawrisch, K. Evidence for specificity in lipid-rhodopsin interactions. J. Biol. Chem. 281, 33233-33241 (2006).

111 Andersen, O. S. \& Koeppe, R. E. 2nd. Bilayer thickness and membrane protein function: an energetic perspective. Annu. Rev. Biophys. Biomol. Struct. 36 107-130 (2007)

112. Sharma, P. et al. Nanoscale organization of multiple $\mathrm{GPI}$-anchored proteins in living cell membranes. Cell 116, 577-589 (2004)

113. Recktenwald, D. J. \& McConnell, H. M. Phase equilibria in binary mixtures of phosphatidylcholine and cholesterol. Biochemistry 20, 4505-4510 (1981)

114. Wang, T. Y. \& Silvius, J. R. Cholesterol does not induce segregation of liquid-ordered domains in bilayers modeling the inner leaflet of the plasma membrane. Biophys. J. 81, 2762-2773 (2001)

115. Kiessling, V., Crane, J. M. \& Tamm, L. K. Transbilayer effects of raft-like lipid domains in asymmetric planar bilayers measured by single molecule tracking. Biophys. J. 91, 3313-3326 (2006).

116. van Meer, G., Halter, D., Sprong, H., Somerharju, P. \& Egmond, M. R. ABC lipid transporters: extruders, flippases, or flopless activators? FEBS Lett. 580, 1171-1177 (2006)

117. Veatch, S. L. \& Keller, S. L. Separation of liquid phases in giant vesicles of ternary mixtures of phospholipids and cholesterol. Biophys. J. 85, 3074-3083 (2003).

118. Veatch, S. L., Polozov, I. V., Gawrisch, K. \& Keller, S. L. Liquid domains in vesicles investigated by NMR and fluorescence microscopy. Biophys. J. 86, 2910-2922 (2004).

119. Wassall, S. R. et al. Order from disorder, corralling cholesterol with chaotic lipids. The role of polyunsaturated lipids in membrane raft formation. Chem. Phys. Lipids 132, 79-88 (2004).

120. Veatch, S. L., Gawrisch, K. \& Keller, S. L. Closed-loop miscibility gap and quantitative tie-lines in ternary membranes containing diphytanoyl PC. Biophys. J. 90, 4428-4436 (2006)

121. Bakht, O., Pathak, P. \& London, E. Effect of the structure of lipids favoring disordered domain formation on the stability of cholesterol-containing ordered domains (lipid rafts): identification of multiple raft-stabilization mechanisms. Biophys. J. 93 , 4307-4318 (2007)

Polyunsaturated acyl chains of membrane lipids can effectively drive the formation of membrane rafts because of especially poor packing with cholesterol.

122. Kucerka, N., Tristram-Nagle, S. \& Nagle, J. F. Closer look at structure of fully hydrated fluid phase DPPC bilayers. Biophys. J. 90, L83-L85 (2006).

123. Liu, K., Hua, Z., Nepute, J. A. \& Graham, T. R. Yeast P4-ATPases Drs $2 p$ and Dnf1 $p$ are essential cargos of the NPFXD/Sla $1 p$ endocytic pathway. Mol. Biol. Cell 18, 487-500 (2007)

DATABASES

UniProtKB: http://beta.uniprot.org/uniprot

CERT |Dnf1 |Dnf2 | Dnf3 |Drs2 | FAPP2 | Lem3 | NIR2 |ORP | Pdr17|Psd2|Sec14|SMS2 |StAR | TSPOIVAP

FURTHER INFORMATION

Lipid MAPS: http://www.lipidmaps.org/data/classification/ index.htm

Gerrit van Meer's homepage: http://www.me.chem.uu.n/ groupmembers/gerrit/vanmeer.html Dennis R. Voelker's homepage: http://www.nationaljewish org/about/phys-fac/detail.aspx?doctorl $\mathrm{D}=83$

Gerald W. Feigenson's homepage: http://www.mbg.cornell. edu/faculty-staff/faculty/feigenson.cfm ALL LINKS ARE ACTIVE IN THE ONLINE PDF 